\title{
Gravity drives the evolution of infrared dark hubs: JVLA observations of SDC13 ${ }^{\star}$
}

\author{
G. M. Williams ${ }^{1}$, N. Peretto ${ }^{1}$, A. Avison ${ }^{2,3}$, A. Duarte-Cabral ${ }^{1}$, and G. A. Fuller ${ }^{2}$ \\ ${ }^{1}$ School of Physics and Astronomy, Cardiff University, Queens Buildings, The Parade, Cardiff CF24 3AA, UK \\ e-mail: williamsgwen12@gmail.com \\ 2 Jodrell Bank Centre for Astrophysics, School of Physics and Astronomy, University of Manchester, Manchester M13 9PL, UK \\ ${ }^{3}$ UK ALMA Regional Centre Node, Manchester M13 9PL, UK
}

Received 17 July 2017 / Accepted 22 January 2018

\begin{abstract}
Context. Converging networks of interstellar filaments, that is hubs, have been recently linked to the formation of stellar clusters and massive stars. Understanding the relationship between the evolution of these systems and the formation of cores and stars inside them is at the heart of current star formation research.

Aims. The goal is to study the kinematic and density structure of the SDC13 prototypical hub at high angular resolution to determine what drives its evolution and fragmentation.

Methods. We have mapped SDC13, a $\sim 1000 M_{\odot}$ infrared dark hub, in $\mathrm{NH}_{3}(1,1)$ and $\mathrm{NH}_{3}(2,2)$ emission lines, with both the Jansky Very Large Array and Green Bank Telescope. The high angular resolution achieved in the combined dataset allowed us to probe scales down to $0.07 \mathrm{pc}$. After fitting the ammonia lines, we computed the integrated intensities, centroid velocities and line widths, along with gas temperatures and $\mathrm{H}_{2}$ column densities.

Results. The mass-per-unit-lengths of all four hub filaments are thermally super-critical, consistent with the presence of tens of gravitationally bound cores identified along them. These cores exhibit a regular separation of $\sim 0.37 \pm 0.16 \mathrm{pc}$ suggesting gravitational instabilities running along these super-critical filaments are responsible for their fragmentation. The observed local increase of the dense gas velocity dispersion towards starless cores is believed to be a consequence of such fragmentation process. Using energy conservation arguments, we estimate that the gravitational to kinetic energy conversion efficiency in the SDC13 cores is $\sim 35 \%$. We see velocity gradient peaks towards $\sim 63 \%$ of cores as expected during the early stages of filament fragmentation. Another clear observational signature is the presence of the most massive cores at the filaments' junction, where the velocity dispersion is largest. We interpret this as the result of the hub morphology generating the largest acceleration gradients near the hub centre.

Conclusions. We propose a scenario for the evolution of the SDC13 hub in which filaments first form as post-shock structures in a supersonic turbulent flow. As a result of the turbulent energy dissipation in the shock, the dense gas within the filaments is initially mostly sub-sonic. Then gravity takes over and starts shaping the evolution of the hub, both fragmenting filaments and pulling the gas towards the centre of the gravitational well. By doing so, gravitational energy is converted into kinetic energy in both local (cores) and global (hub centre) potential well minima. Furthermore, the generation of larger gravitational acceleration gradients at the filament junctions promotes the formation of more massive cores.
\end{abstract}

Key words. stars: formation - stars: massive - ISM: clouds - ISM: kinematics and dynamics - ISM: structure

\section{Introduction}

The star formation process can be perceived as the mechanism leading to the concentration of diffuse interstellar clouds into compact, nuclear burning, balls of gas. While the importance of interstellar filaments had been recognised already in the seventies (Schneider \& Elmegreen 1979), observations of the Galactic interstellar medium with Herschel have revealed that they are a key intermediate stage towards the formation of stars (André et al. 2010; Molinari et al. 2010; Arzoumanian et al. 2011). Indeed, the majority of pre-stellar and proto-stellar cores are embedded in thermally supercritical filaments (Polychroni et al. 2013; Könyves et al. 2015; Marsh et al. 2016), that is filaments whose mass-per-unit-length $M_{\text {line }}$ is larger than the theoretical upper limit for an isothermal, infinitely long cylinder to maintain hydrostatic equilibrium (Ostriker 1964). Understanding the

\footnotetext{
* The FITS files of the JVLA and GBT combined $\mathrm{NH}_{3}(1,1)$ and $\mathrm{NH}_{3}(2,2)$ data cubes are also available at the CDS via anonymous ftp to cdsarc.u-strasbg.fr (130.79.128.5) or via http://cdsarc.u-strasbg.fr/viz-bin/qcat?]/A+A/613/A11
}

connection between filament evolution and core formation has become one of the main goals of star formation research over the past decade.

While a lot of effort has focused on the properties of individual filaments in nearby star-forming regions (Arzoumanian et al. 2011; Peretto et al. 2012; Palmeirim et al. 2013; Panopoulou et al. 2014; Salji et al. 2015), here we focus on hub filament systems (HFS), i.e. a small network of spatially converging interstellar filaments (Myers 2009). The converging nature of such systems is suggestive of the role played by gravity in shaping them. Follow-up observations have shown that hubs are likely collapsing on parsec scales, gathering matter at their centre as a result of the collapse (Kirk et al. 2013; Peretto et al. 2013, 2014; Liu et al. 2013). One of the most massive protostellar cores ever observed in the Galaxy has been found lying at the centre of such hubs (Peretto et al. 2013), indicating that they might play a key role in the formation of massive stars. Schneider et al. (2012) also showed that stellar protoclusters in the Rosette molecular cloud tend to form at the junction of filamentary structures. Understanding how hubs form and evolve can tell 
us what physical mechanisms are behind the fragmentation of filaments and how their interaction influence the formation of more massive objects. High-angular resolution observations of infrared dark clouds (IRDCs) provides us with the opportunity to study such systems very early on in their evolution.

IRDCs are cold $(T<20 \mathrm{~K})$ and dense $\left(n>10^{3} \mathrm{~cm}^{-3}\right)$ reservoirs of gas seen in extinction against the bright mid-infrared emission of the Galactic plane background (Perault et al. 1996; Egan et al. 1998; Simon et al. 2006; Peretto \& Fuller 2009; Butler \& Tan 2009; Peretto et al. 2010). They are considered to be mostly undisturbed by stellar feedback due to the lack of a significant embedded population of stars, and exhibit a wide range of morphologies, masses and sizes (Peretto et al. 2010). The most massive of these IRDCs are thought to contain the initial conditions for massive star formation (Rathborne et al. 2006; Pillai et al. 2006; Beuther \& Steinacker 2007).

SDC13 (Fig. 1) is a remarkable filamentary hub IRDC system that lies $3.6 \pm 0.4 \mathrm{kpc}$ away in the Galactic plane and contains $\sim 1000 M_{\odot}$ of material (Peretto et al. 2014). Each of its four well-defined parsec-long filaments (including SDC13.174-0.07, SDC13.158-0.073 and SDC13.194-0.073, Peretto \& Fuller 2009, subsequently named following their onsky orientation) converges on a central hub. With the analysis of $1.2 \mathrm{~mm}$ dust continuum data from the MAMBO bolometer array on the IRAM $30 \mathrm{~m}$ telescope (at $12^{\prime \prime}$ resolution) 18 compact sources were extracted and their starless or proto-stellar nature identified from $8 \mu \mathrm{m}$ and $24 \mu \mathrm{m}$ Spitzer images. The two most massive cores (named MM1 and MM2) are located right at the junction of the hub filaments, the substructure of which was studied using high resolution $\left(<3^{\prime \prime}\right) 1.3 \mathrm{~mm}$ SMA dust continuum emission (McGuire et al. 2016). MM1 was found to contain two subfragments, the centremost being brighter than the MM2 fragment. Modelling the cores with RADMC-3D reveals that MM2 requires a steeper density profile than MM1, suggesting that it may be most likely to form a single massive central object (Girichidis et al. 2011). Tracing the dense gas in $\mathrm{N}_{2} \mathrm{H}^{+}(1-0)$ with the IRAM $30 \mathrm{~m}$ telescope (at $27^{\prime \prime}$ resolution) Peretto et al. (2014) identified velocity gradients along each of the filamentary arms of SDC13. Infall velocities larger at the filament ends anti-correlate with the velocity dispersion gradients which reach their maximum at the hub centre. This was interpreted as the consequence of the collapse of the gas along the filaments, generating an increase of kinetic pressure at the centre of the hub and providing the necessary conditions for the formation of super-Jeans cores, i.e. cores with masses that are several times larger than the local Jeans mass.

In this paper we present new high-resolution ammonia observations of SDC13 obtained with the Jansky Very Large Array (JVLA) and the Green Bank Telescope (GBT). These observations allow us to resolve, for the first time, the density structure and kinematics of the SDC13 filaments, expanding on the analysis of previous work. In Sect. 2 we present the observations and discuss the process of combining the two data sets. Section 3 presents our line fitting and analysis of the observed kinematics of the system. In Sect. 4 we discuss the identification of filament and core structures. We discuss the fragmentation and dynamics of the system in Sect. 5, and finally, we summarise our conclusions in Sect. 6.

\section{Observations}

We observed ammonia $\left(\mathrm{NH}_{3}\right)$ at the position of SDC13 with both the JVLA and the GBT. $\mathrm{NH}_{3}$ is rather abundant in star

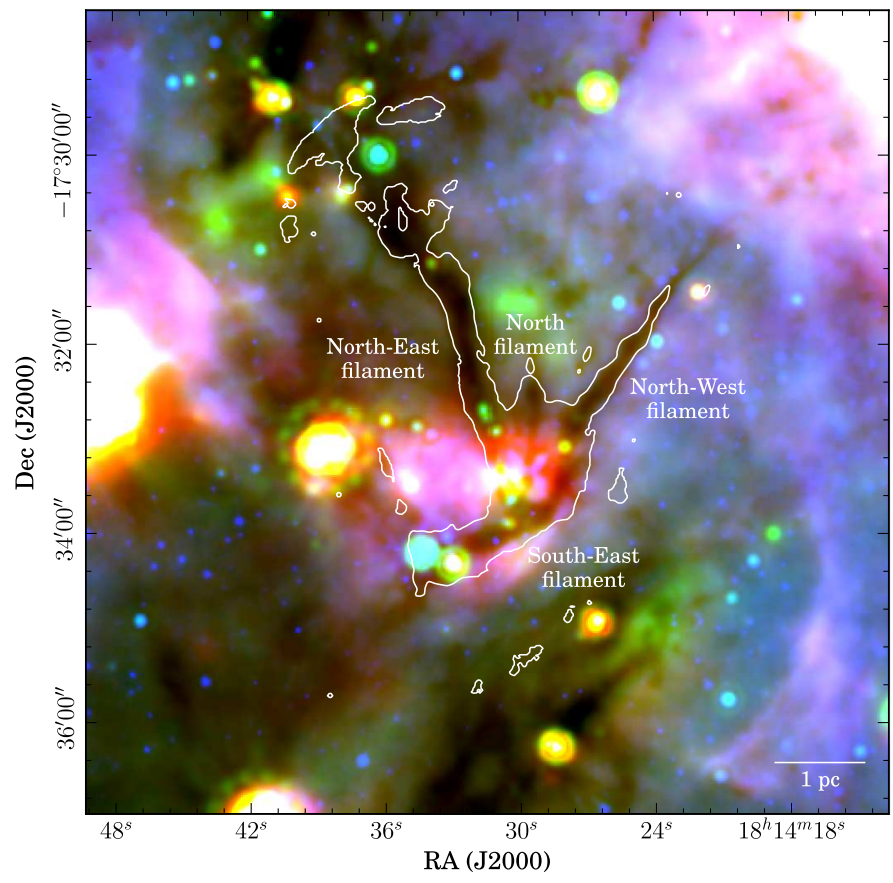

Fig. 1. Three colour image of SDC13. $R, G$ and $B$ bands correspond to $70 \mu \mathrm{m}$ HIGAL (Molinari et al. 2010), $24 \mu \mathrm{m}$ Spitzer MIPSGAL (Carey et al. 2009) and $8 \mu \mathrm{m}$ Spitzer GLIMPSE (Churchwell et al. 2009) maps, respectively. The overplotted contour is from the $\mathrm{H}_{2}$ column density map at $2 \times 10^{22} \mathrm{~cm}^{-2}$ (see Sect. 3.2 and the middle panel of Fig. 5). A 1 pc scale bar is plotted in the bottom right corner. The filament names are labelled following those used by Peretto et al. (2014).

forming regions (Cheung et al. 1968), with an abundance of $\left[\mathrm{NH}_{3}\right] /\left[\mathrm{H}_{2}\right] \sim 3 \times 10^{-8}$ (Harju et al. 1993). The $(J, K)=(1,1)$ and $(2,2)$ rotation inversion transitions with their relatively large critical density $\left(n_{\text {crit }}=10^{3} \mathrm{~cm}^{-3}\right.$, Ho \& Townes 1983; Shirley 2015) are particularly good tracers of the cold and dense gas during the early stages of star formation as they are excited at the low temperatures $(<20 \mathrm{~K})$ of molecular clouds and IRDCs, and exhibit very little, if any, depletion. Studies of the hyperfine splitting of the $(1,1)$ and $(2,2)$ transitions (e.g. Gunther-Mohr 1954; Kukolich 1967) show that they have 18 and 24 hyperfine components, emitting in the radio regime at 23.69 and $23.72 \mathrm{GHz}$, respectively (e.g. Ho \& Townes 1983). The detection of both these lower metastable states allows for the direct calculation of the opacity, temperature and column density of the gas (e.g. Ho \& Townes 1983; Ungerechts et al. 1986).

\subsection{JVLA observations}

The $\mathrm{NH}_{3}(1,1)$ and $\mathrm{NH}_{3}(2,2)$ transitions were observed at the position of the SDC13 infrared dark cloud (J2000 18:14:30.0$17: 32: 50.0)$ at $23.7 \mathrm{GHz}$ using the JVLA in the DnC configuration, using the $1 \mathrm{GHz}$ band with $4 \mathrm{MHz}$ sub-bands. We observed the $\mathrm{NH}_{3}(3,3)$ transition, but it was not significantly detected. The angular resolution achieved of $3.3^{\prime \prime}$ is an 8-fold improvement on the $27^{\prime \prime}$ resolution of the $\mathrm{N}_{2} \mathrm{H}^{+}(1-0)$ molecular line IRAM $30 \mathrm{~m}$ data (Peretto et al. 2014), probing 0.07 pc spatial scales. A mosaic across the full $5^{\prime} \times 5^{\prime}$ extent of the cloud was performed in eight, half-beam spaced pointings, collected over 8 observing sessions between 18 and 20 of May 2013, with 102.4 min integration time per position. Further information on these data are provided in Table 1. 
Table 1. Summary of the observational properties of the JVLA, GBT and combined data.

\begin{tabular}{lcccccc}
\hline \multicolumn{1}{c}{ Telescope } & \multicolumn{2}{c}{ JVLA } & \multicolumn{2}{c}{ GBT } & \multicolumn{2}{c}{ Combined data } \\
\hline Parameters & $\mathrm{NH}_{3}(1,1)$ & $\mathrm{NH}_{3}(2,2)$ & $\mathrm{NH}_{3}(1,1)$ & $\mathrm{NH}_{3}(2,2)$ & $\mathrm{NH}_{3}(1,1)$ & $\mathrm{NH}_{3}(2,2)$ \\
\hline Channel width $(\mathrm{kHz})$ & 3.906 & 3.906 & 2.86 & 2.86 & 7.812 & 7.812 \\
Velocity resolution $\left(\mathrm{km} \mathrm{s}^{-1}\right)$ & 0.049 & 0.049 & 0.036 & 0.036 & 0.098 & 0.098 \\
RMS noise (mJy beam $\left.{ }^{-1}\right)$ & 3.53 & 3.63 & 170.0 & 180.0 & 3.71 & 3.82 \\
Beam major axis (") & 3.87 & 3.77 & 31.32 & 31.32 & 4.35 & 4.30 \\
Beam minor axis (") & 2.81 & 2.87 & 31.32 & 31.32 & 3.44 & 3.48 \\
Position angle $\left(^{\circ}\right)$ & 30.95 & 30.56 & 0.0 & 0.0 & 30.95 & 30.56 \\
\hline
\end{tabular}

Notes. For the purpose of hyperfine structure fitting, velocity resolutions were smoothed to $0.098 \mathrm{~km} \mathrm{~s}^{-1}$ for the JVLA, and $0.072 \mathrm{~km} \mathrm{~s}^{-1}$ for the GBT. RMS noise was estimated from emission free areas. The combined data were smoothed by a Gaussian kernel of $2.4^{\prime \prime}$, and have the same spectral resolution as the smoothed JVLA data. The quoted RMS noise of the GBT data includes the applied calibration factor.

Data reduction and calibration was completed using $\mathrm{CASA}^{1}$ (McMullin et al. 1992). Phase and flux calibration was completed using the two bright quasars J1832-1035 and 3C286, respectively. The phase calibrator was chosen to sit within $6^{\circ}$ to the target on the sky to ensure similar atmospheric conditions. Bandpass calibration was completed with the J1743-0350 quasar. We flagged any bad data using the flagcmd() and flagdata() tasks within CASA, and imaged both $\mathrm{NH}_{3}$ transitions using the deconvolution task, clean(). Our imaging implements the Natural weighting scheme, which puts less emphasis on the smallest scale coverage of the data. This was done as our $u-v$ coverage was slightly stretched in this regime due to the low declination of the source.

\subsection{GBT observations}

We used the 7-beam $K$-band Focal Plane Array at the GBT with the VEGAS (Versatile GBT Astronomical Spectrometer) backend to observe the $\mathrm{NH}_{3}(1,1)$ and $(2,2)$ transitions employing a position switched scheme, where the Off source reference position (18:13:59.3-17:35:02.0) was devoid of $\mathrm{NH}_{3}$ emission. Two maps were scanned in right ascension, while another two were scanned in declination so as to mitigate any artefacts that could arise during imaging had only one mapping scheme been used. All four maps were observed on 14 November 2015, each with an integration time of $17.9 \mathrm{~min}$ (see Table 1 for data properties). We ran the GBT pipeline (Masters et al. 2011) on each map scan separately to calibrate, reduce and subtract the continuum emission. All scans were then combined and imaged in AIPS using the aipspy ParselTongue scripts included with the pipeline. Possible issues with the noise diode voltages at the time of observation skewed our flux calibration but had no effect on the quality of the data. This resulted in the measured flux of the flux calibrator (J1833-2103, a quasar) to be lower than expected from published calibration tables. We applied an appropriate calibration factor (a ratio of the expected to the measured flux) to our data to correct for this.

\subsection{Combination of JVLA and GBT data}

Interferometers only probe a limited range of spatial frequencies of the source brightness spatial distribution, the largest frequency being determined by the shortest distance between

\footnotetext{
1 The Common Astronomy Software Applications package http://casa.nrao.edu
}

any pair of the interferometer antennas. As a consequence, interferometers filter out extended emission. In order to recover this large-scale emission, and therefore be able to analyse the source structure on all spatial scales, one needs to combine interferometer data with single dish data. Hence, we combined our JVLA data with the GBT observations.

As a preliminary combination step, we re-clean our JVLA visibilities in the same way as described in Sect. 2.1, whilst using the GBT cube as a starting model (Dirienzo et al. 2015). This method assists in initializing the clean algorithm by using the GBT primary beam to determine the flux scale. Moreover, as the JVLA is missing information at the shortest baselines, this approach allows the clean algorithm to extrapolate the GBT data and converge on a better solution in this $u-v$ regime. We note that this is not the same as performing a joint deconvolution.

Combination is achieved with the use of the CASA task, feather. With the high and low resolution images as inputs, feather Fourier transforms the two cubes, applies a low-pass filter to the low resolution data and a high-pass filter to the high resolution data, sums the two Fourier and filtered cubes in $u-v$ space, and reconvolves the final combined cube. The low and high pass filters (weights calculated from the input clean beams) are applied to recover the larger scale emission of the GBT data at low $u-v$ distances which the JVLA naturally filters out, whilst retaining the fine scale structure probed by the JVLA at larger $u-$ $v$ distances which the GBT cannot be sensitive to. The primary beam response of the JVLA was applied to the GBT data prior to feathering, so that only emission within the JVLA mosaic area was considered.

The casafeather visual interface provides the tools to inspect slices of the $u-v$ plane of both the original and weighted deconvolved input images. This is especially useful while setting the sdfactor parameter, which applies a flux scaling factor to the low resolution image. For the conservation of flux, one would expect the fluxes of the weighted deconvolved high and low resolution images to be roughly equal. One can achieve this by altering the sdfactor until the casafeather plots demonstrate this equality. Given the uncertainty in the flux calibration of the GBT, we expected a factor would be required, however, we find a satisfactory result with the default sdfactor of 1 . This demonstrates that the calibration factor applied to the GBT data in the first instance was sound and appropriate.

Figure 2 shows a comparison of the GBT, JVLA and combined integrated intensity maps of $\mathrm{NH}_{3}(1,1)$. It is clear that the combined data recovers more extended flux than the JVLA-only, resulting in a doubling of the JVLA flux on the 


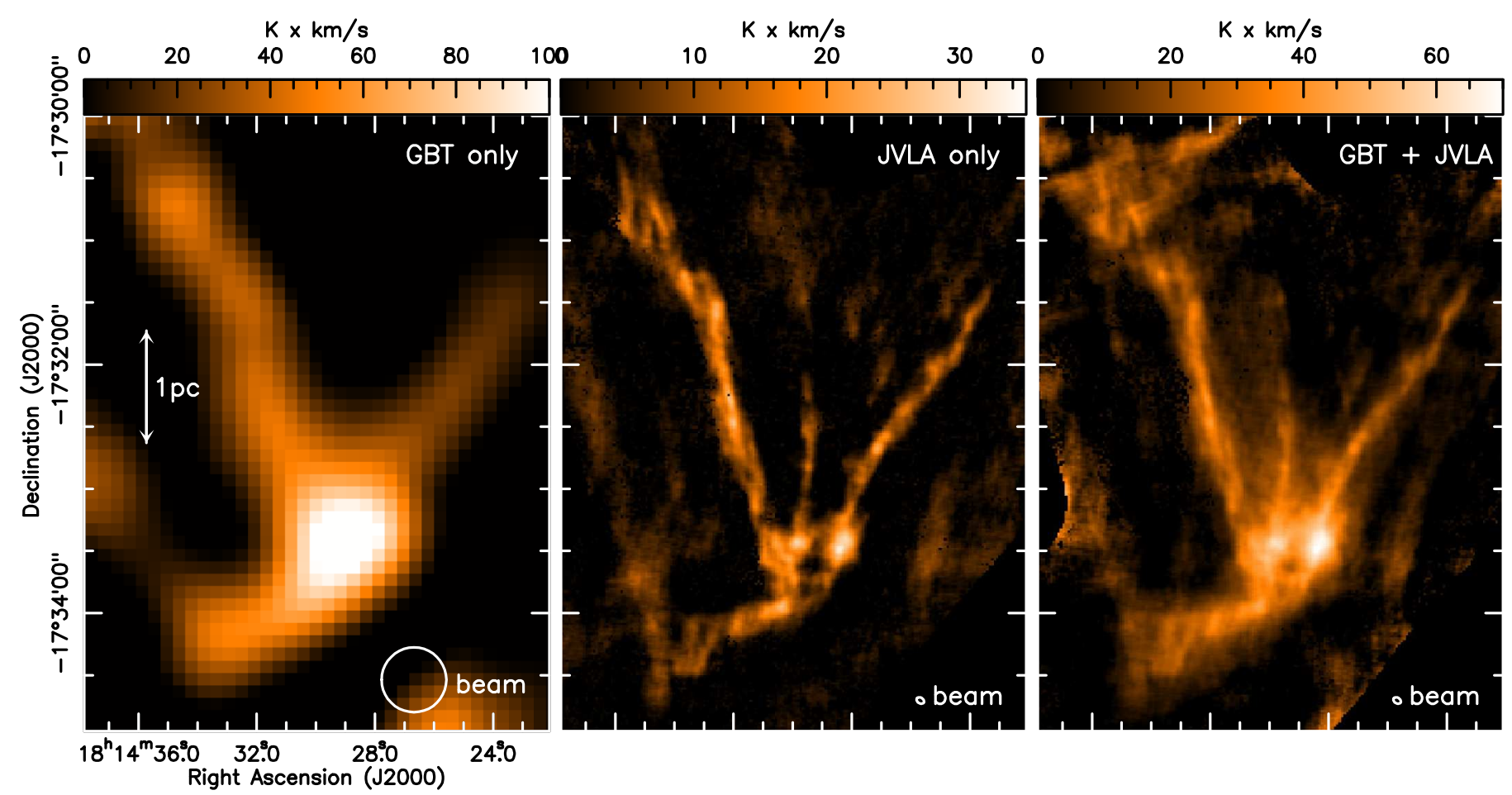

Fig. 2. Comparison between the integrated intensity maps of the $\mathrm{NH}_{3}(1,1)$ emission from the GBT (left), the JVLA (middle) and the combined GBT and JVLA (right) data sets. The GBT map displayed here has already had the calibration factor applied (see Sect. 2.2). The beam sizes are plotted in the bottom right corner of each panel. A parsec scale at the distance of the cloud is plotted in the first panel.

whole, whilst retaining the small scale detail and RMS noise of the interferometer. We can clearly see that the negative bowl features, a consequence of missing flux, that surrounds the cloud structure in the JVLA-only map are mostly eradicated in the combined map and filled by the recovered extended emission. A check was made on the conservation of flux, performed by smoothing the feathered image to the resolution of the GBT, converting the units to Jy per GBT beam, and dividing by the original GBT map. In doing this, we find that flux is conserved. In the filament regions, we find a ratio of unity on the whole, whilst in the regions of significant emission such as the hub the combined flux is typically $\sim 20 \%$ less than the GBT map (a similar result to that reported by Dirienzo et al. 2015).

In order to gain in signal to noise ratio we convolved the combined datacube with a Gaussian kernel of 2.4", degrading the angular resolution to $\sim 4^{\prime \prime}$.

\section{Results}

Here we present the results obtained from fitting the $\mathrm{NH}_{3}$ hyperfine structure, and from deriving a $\mathrm{H}_{2}$ column density map from the $\mathrm{NH}_{3}$ emission.

\subsection{Line fitting}

We performed hyperfine structure fitting of the combined JVLA and GBT data using the $\mathrm{NH}_{3}(1,1)$ and $\mathrm{NH}_{3}(2,2)$ fitting schemes within the CLASS ${ }^{2}$ software, where the spectra are fitted at every pixel over all channels. Figure 3 shows examples of ten spectra and their hyperfine structure fits from positions distributed across the cloud. After visual inspection, we do not find multiple

2 The fitting procedure of hyperfine structure is described at: https://www.iram.fr/IRAMFR/GILDAS/doc/pdf/class.pdf velocity components, even at the filament junctions in the hub centre, therefore we proceed to fit a single velocity component everywhere. Using the result command, a model fit to the data was created, where opacity-corrected Gaussian profiles are fitted to each hyperfine transition. The channel maps of the resulting cube are shown in Fig. A.1. We use these model fit cubes for all analysis conducted in the rest of the paper.

Due to a miss-alignment of the centre of the bandwidth with respect to the main hyperfine component at the cloud velocity $\left(v_{\text {sys }}=37 \mathrm{~km} \mathrm{~s}^{-1}\right)$ we do not detect the fifth hyperfine satellite of the $\mathrm{NH}_{3}(1,1)$ line at $57 \mathrm{~km} \mathrm{~s}^{-1}$. To correct for this missing flux, in the construction of integrated intensity maps we assumed that the missing component's intensity was identical to that of its symmetric pair at $17 \mathrm{~km} \mathrm{~s}^{-1}$. Using the GBT data, which does cover all hyperfine components, we estimate that this correction leads to an uncertainty on the total integrated intensity of $\leq 5 \%$.

\subsubsection{Integrated intensity}

Figure 4 (left) shows the integrated intensity of the $\mathrm{NH}_{3}(1,1)$ (top) and $\mathrm{NH}_{3}(2,2)$ (bottom) hyperfine structure lines. The structures in both maps are very similar even though, being typically 6 times weaker, the $\mathrm{NH}_{3}(2,2)$ emission is less extended and noisier than the $\mathrm{NH}_{3}(1,1)$ emission. When comparing the integrated intensity maps (or column density map - see Sect. 3.2) to both the $1.2 \mathrm{~mm}$ dust continuum and $8 \mu \mathrm{m}$ maps (see Fig. 5) we find excellent agreement between the structures seen in the different images. All four filamentary arms seen in dust extinction/emission are well resolved in our $\mathrm{NH}_{3}$ maps. This demonstrates that ammonia is an excellent tracer of cold dense gas as numerous previous works had already theoretically (Bergin \& Langer 1997) or observationally (Lu et al. 2014) shown. There is, however, one exception to this excellent match between ammonia and dust emission. Indeed, we do not detect MM1 
G. M. Williams et al.: Gravity drives the evolution of infrared dark hubs: JVLA observations of SDC13

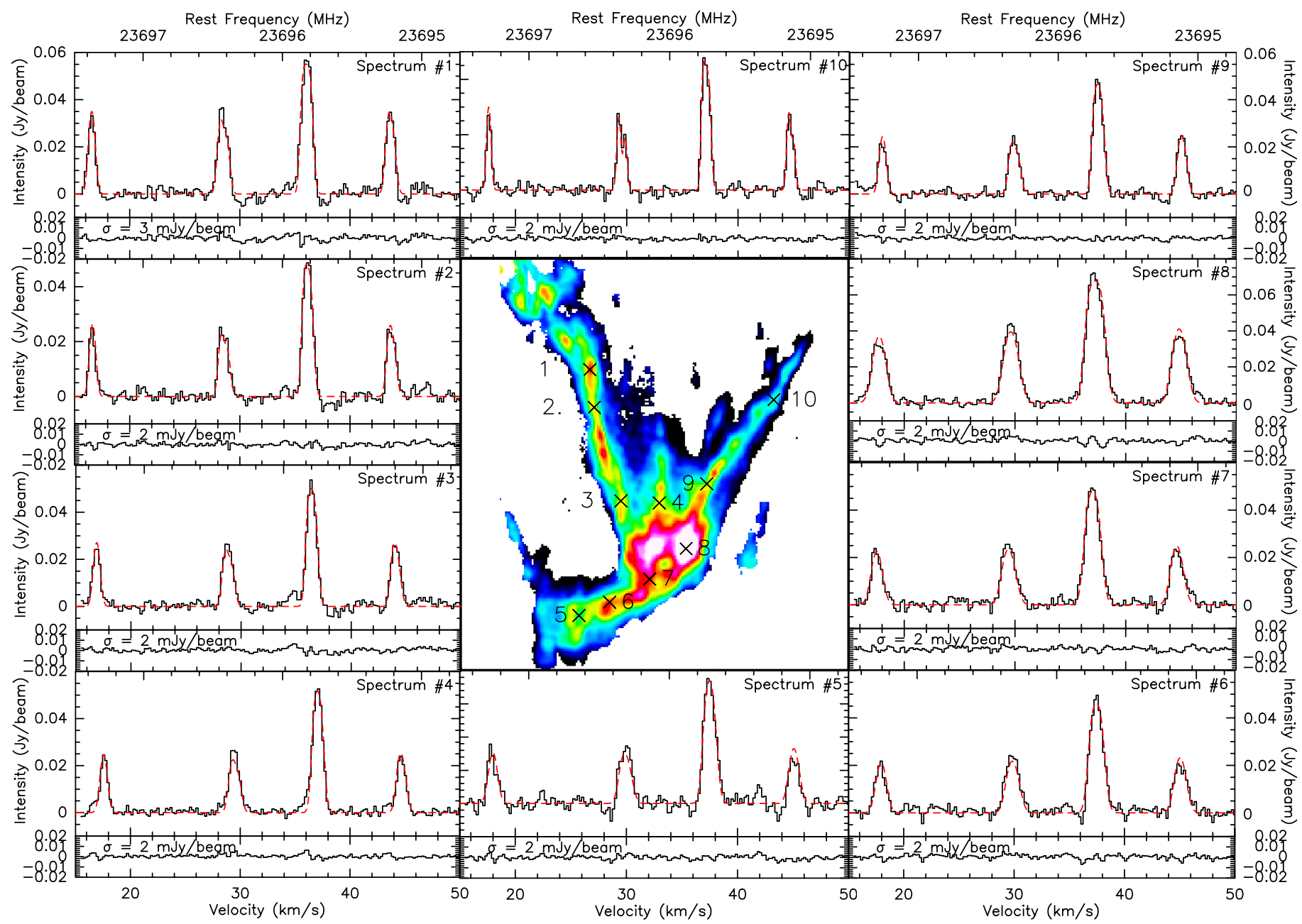

Fig. 3. Examples of $\mathrm{NH}_{3}(1,1)$ spectra at ten positions in the cloud, plotted surrounding the $\mathrm{H}_{2}$ column density derived from the $\mathrm{NH}_{3}$ emission (discussed later in Sect. 3.2). The model fit is plotted over each spectrum in red, whilst the residual of the two is plotted in the bottom panel with the standard deviation quoted in $\mathrm{mJy}_{\text {beam }}{ }^{-1}$. Regions of specific interest include those where the velocity width is seen to increase (spectra 1 and 6), positions where the centroid velocity changes as a filament meets the central hub (spectra 3,4 and 7) and the large starless core MM2 (spectrum 8). It is clear that there is only a single velocity component everywhere in the cloud, with some lines being narrow enough to start resolving further hyperfine components (spectrum 10), and some showing the tell-tale signs of the cloud identified by Peretto et al. (2014) at a $V_{\text {sys }}=54 \mathrm{kms}^{-1}$ that likely overlaps in projection with the north-east filament (spectrum 1 and 2).

$\left(74.8 \pm 27.1 M_{\odot}\right.$ within $0.26 \mathrm{pc}$, Peretto et al. 2014 , labelled in Fig. 5), the largest proto-stellar source detected in the $1.2 \mathrm{~mm}$ dust continuum map. This is a rather striking result since ammonia has consistently shown to be a good tracer of both cold and warm dense gas in star-forming clouds (Pillai et al. 2011; Ragan et al. 2011). Because of the proto-stellar nature of MM1, the lack of ammonia in the gas phase points towards a destruction mechanism rather than depletion. Chemical modelling of MM1 would be necessary to better constrain the physical origin of the lack of ammonia, but this goes beyond the scope of this paper. Also, the cavity in column density (centred on 18:14:29.3-17:33:37.4) is also seen in the $8 \mu \mathrm{m}$ Spitzer data as diffuse emission, with no evidence of any related compact source that may have caused clearing of surrounding material.

\subsubsection{Centroid velocity}

The channel map of the main $\mathrm{NH}_{3}(1,1)$ transition in this model cube is shown in Fig. A.1. It shows large-scale velocity structures, with filaments appearing at different velocities, with a total velocity span of $\sim 7 \mathrm{~km} \mathrm{~s}^{-1}$, consistent with the global velocity structure observed in $\mathrm{N}_{2} \mathrm{H}^{+}(1-0)$ by Peretto et al. (2014). Figure 4 (middle columns) display the line of sight centroid velocity from both the $\mathrm{NH}_{3}(1,1)$ and $(2,2)$ transitions. As a result of the increased angular resolution (almost by an order of magnitude), we observe a far more complex velocity field in SDC13 than previously revealed by the IRAM $30 \mathrm{~m}$ data. In particular, we resolve both longitudinal and radial velocity gradients across all filaments (where filaments are named following the same convention as Peretto et al. 2014, and their extraction is discussed in Sect. 4.2). This is most evident across the bottom half of the north-east orientated filament, and across the entire south-east orientated filament (labelled in Figs. 4 and 5). At face value, it is not clear that our ammonia velocity map is consistent with the $\mathrm{N}_{2} \mathrm{H}^{+}(1-0)$ data published by Peretto et al. (2014). In order to check for consistency, we fitted the hyperfine components of the $\mathrm{NH}_{3}(1,1)$ and $\mathrm{NH}_{3}(2,2)$ emission from the GBT data only (see Appendix D), at a similar angular resolution to the IRAM $30 \mathrm{~m}$ data. The GBT velocity map reveals gradients along each and every filamentary arm towards the centre of the system, identical to those observed in the $\mathrm{N}_{2} \mathrm{H}^{+}(1-0)$ IRAM $30 \mathrm{~m}$ data. The 

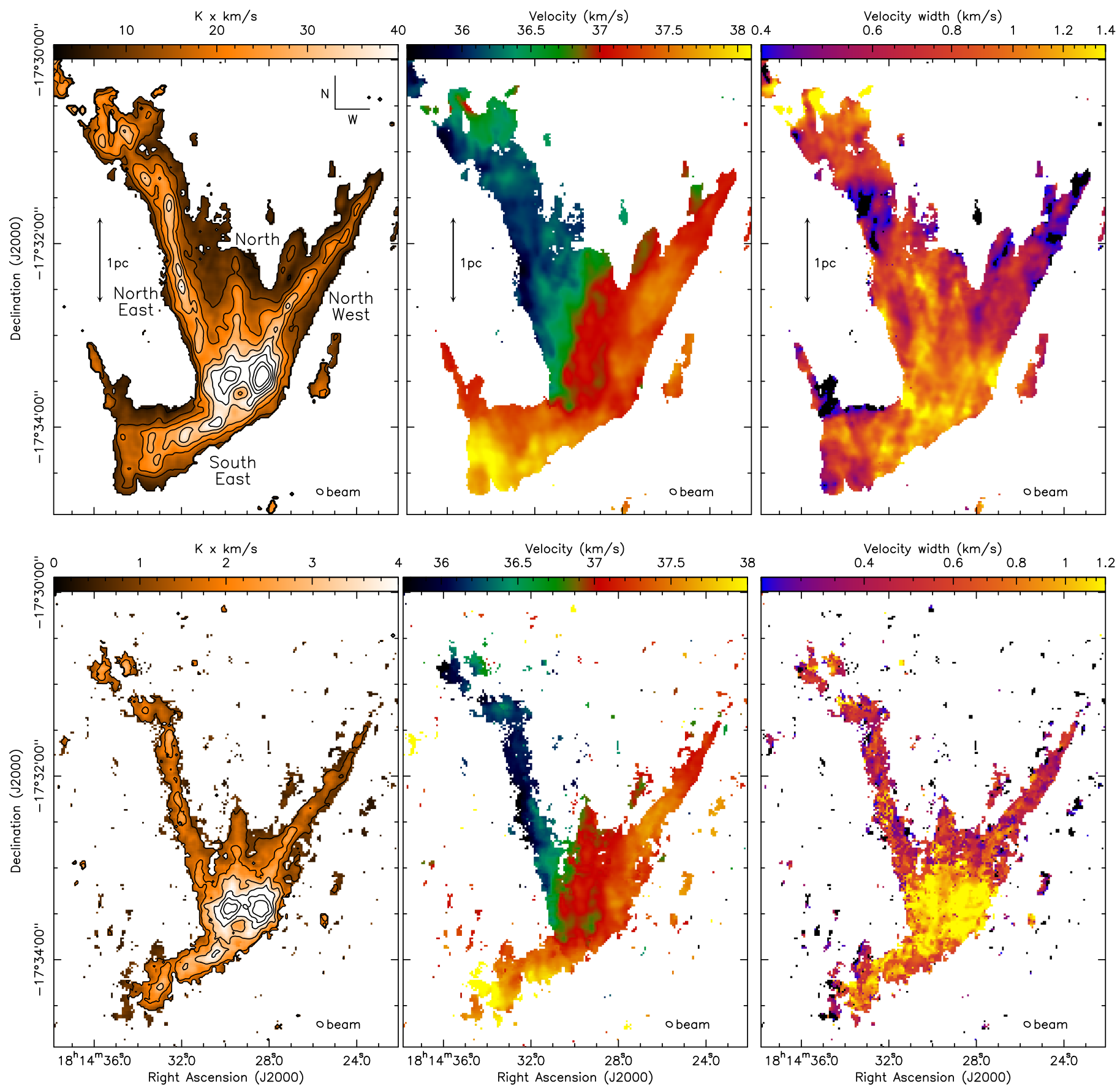

Fig. 4. Integrated intensity (first column), centroid velocity (middle column) and velocity width (right column) of the $\mathrm{NH}_{3}(1,1)$ transition (top row) and $\mathrm{NH}_{3}(2,2)$ transition (bottom row). The $\mathrm{NH}_{3}(1,1)$ data were masked to $5 \sigma$, while the weaker $\mathrm{NH}_{3}(2,2)$ data were masked to $3 \sigma$. Filaments are named in the top left panel. Contours in the top left panel are in steps of $7 \mathrm{~K} \times \mathrm{km} \mathrm{s}^{-1}$, from $5 \mathrm{~K} \times \mathrm{km} \mathrm{s}^{-1}$ to $61 \mathrm{~K} \times \mathrm{km} \mathrm{s}^{-1}$, while contours in the bottom left panel are in steps of $1 \mathrm{~K} \times \mathrm{km} \mathrm{s}^{-1}$, from $1 \mathrm{~K} \times \mathrm{km} \mathrm{s}^{-1}$ to $6 \mathrm{~K} \times \mathrm{km} \mathrm{s}^{-1}$. Beam information is plotted in the bottom right corner of each panel, while the scale of $1 \mathrm{pc}$ is plotted in each panel of the top row.

large number of $\mathrm{NH}_{3}(1,1)$ hyperfine components makes the best fit centroid velocity and velocity width both very accurate. We estimate that the related uncertainty is less than half a channel width, i.e. $\sim 0.05 \mathrm{~km} \mathrm{~s}^{-1}$.

\subsubsection{Velocity width}

Figure 4 (right column) shows the FWHM velocity width of the dense gas. We report an increased velocity width at the filament hub junction, peaking at $\sim 1.6 \mathrm{~km} \mathrm{~s}^{-1}$, consistent with the broadening reported by Peretto et al. (2014). Interestingly, we also observe local increases of the velocity width sprinkled along each filamentary arms. As discussed later in Sect. 4, these are correlated to peaks in the $\mathrm{H}_{2}$ column density. We note that opacity broadening is accounted for as a consequence of the fitting procedure used. However, because of the relatively large peak $\mathrm{NH}_{3}(1,1)$ main line opacity of 4.7 , we independently performed a Gaussian fit of the optically thinner of the hyperfine components. By doing so, we observe the same increase of the line width. We conclude that the line widths plotted in Fig. 4 


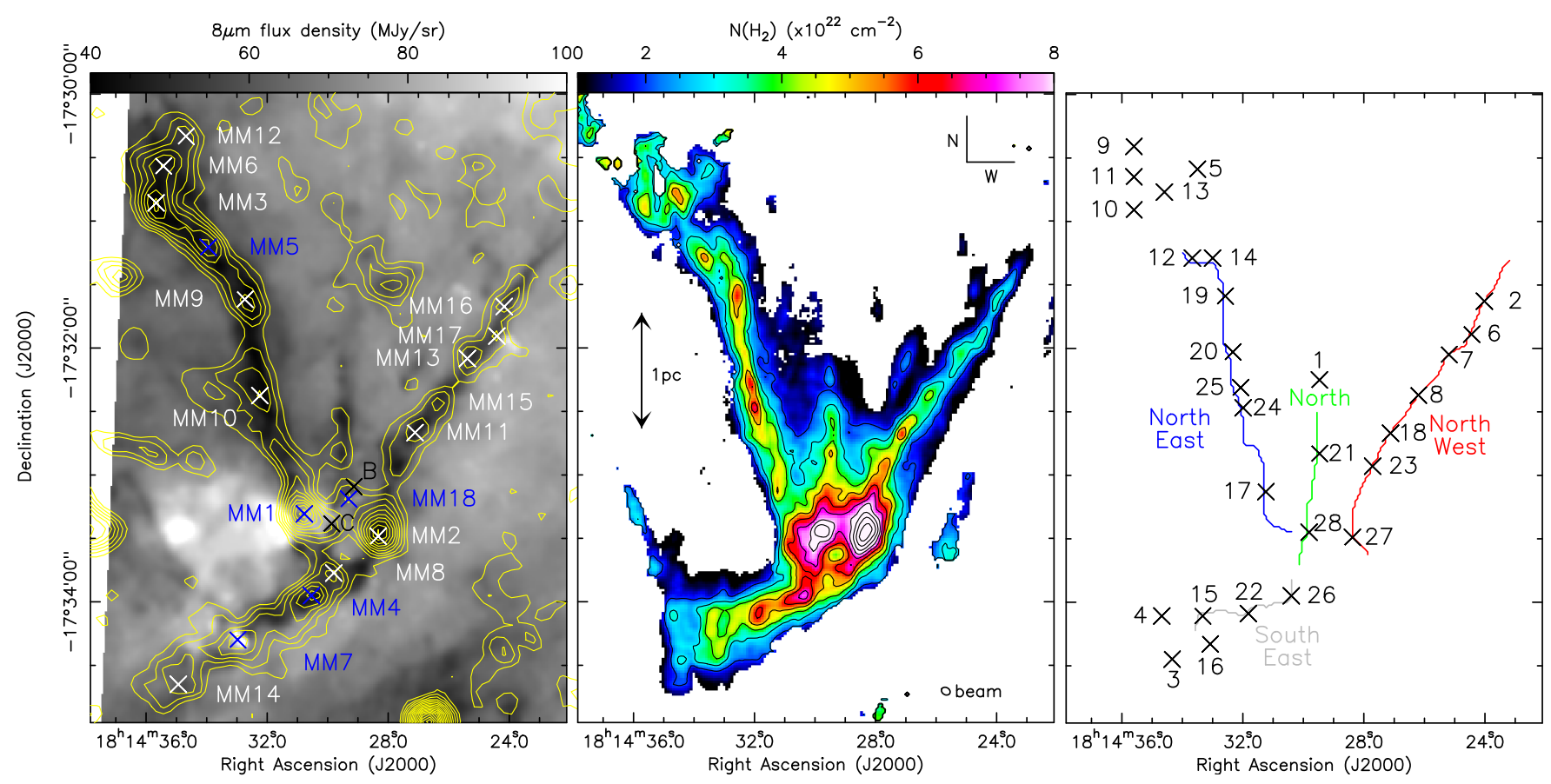

Fig. 5. Left: Spitzer $8 \mu \mathrm{m}$ flux density in units of $\mathrm{MJy} \mathrm{s}^{-1}$, overlaid with IRAM 30m MAMBO $1.2 \mathrm{~mm}$ dust continuum contours in steps of $5 \mathrm{mJy}_{\text {beam }}{ }^{-1}$, from 3 to $88 \mathrm{mJy}_{\text {beam }}{ }^{-1}$. Crosses denote the positions of identified $1.2 \mathrm{~mm}$ MAMBO compact sources (white for starless and blue for proto-stellar, Peretto et al. 2014) and two $1.3 \mathrm{~mm}$ SMA continuum sources (in black crosses, McGuire et al. 2016). Middle: $\mathrm{H}_{2}$ column density map in units of $10^{22} \mathrm{~cm}^{-2}$, derived from the $\mathrm{NH}_{3}$ integrated intensity. Overlaid contours are placed in $1 \times 10^{22} \mathrm{~cm}^{-2}$ steps, from $2 \times 10^{22} \mathrm{~cm}^{-2}$ to $12 \times 10^{22} \mathrm{~cm}^{-2}$. Right: extracted cores (black crosses, numbered according to Table 2) and filaments (labelled coloured lines). The extraction of these structures are discussed in Sect. 4. Extra cores at the outskirts of the north-east and south-east arms are in the more diffuse regions of the cloud where the spines did not extend through. Spine colours match those in subsequent figures.

represent the true velocity dispersion of the dense gas. Furthermore, we also notice the velocity width increases around some localised regions at the edges of filaments, most evident on the Eastern edge of the lower portion of the north-east filament, and the western edge of the lower portion of the north-west filament.

\section{2. $\mathrm{H}_{2}$ column density map}

Ammonia is often called the thermometer of molecular clouds (e.g. Maret et al. 2009), as the temperature of the gas can be directly calculated from the ratio of the intensities of the two main lower metastable transitions, $\mathrm{NH}_{3}(1,1)$ and $\mathrm{NH}_{3}(2,2)$. In turn, the ammonia column density map can be calculated using the temperature, opacity and brightness temperature maps. The derivations of each of these quantities can be found in Appendix B. Using a constant abundance of ammonia with respect to $\mathrm{H}_{2}$ of $3 \times 10^{-8}$ (Harju et al. 1993) one can then compute the $\mathrm{H}_{2}$ column density map of SDC13. This calculation is however limited by the weaker emission of the $\mathrm{NH}_{3}(2,2)$ transition, which in our case traces $42 \%$ less of the cloud area than the $\mathrm{NH}_{3}(1,1)$ transition. To overcome this, we take a median value of the temperature across the cloud of $12.7 \mathrm{~K}$ (the full temperature map and histogram are shown in Appendix B). The corresponding column density map is displayed in Fig. 5. Comparing this column density map to that calculated with a non-uniform temperature, we are satisfied that assuming a constant temperature across the entire cloud does not introduce any significant changes to the morphology of features in the map, whilst extending the coverage of the map (a result also reported by Morgan et al. 2013). Increasing the temperature by a standard deviation $(1.8 \mathrm{~K})$ decreases the column density by $12 \%$. It is important to note that given our non-detection of the MM1 protostellar core, our assumption of constant abundance across the entire cloud (whilst a fair assumption given the excellent correlation between the $\mathrm{NH}_{3}(1,1)$ integrated intensity and the $8 \mu \mathrm{m}$ Spitzer emission) does not hold entirely for such portions of the cloud as the MM1 region. Deriving the $\mathrm{H}_{2}$ column density from the $1.2 \mathrm{~mm}$ dust continuum emission (presented in Peretto et al. 2014) and comparing it to the $\mathrm{NH}_{3}$ column density shows that the assumed abundance is consistent with the data, and has a dispersion of only $\sim 3 \%$. Overall, we take a conservative estimate of the uncertainty on the column density of $20 \%$.

\section{Analysis: structure extraction}

In this section, we discuss the identification of cores and filaments within SDC13.

\subsection{Cores}

Dendrograms are a useful tool for the understanding of hierarchical structure and are invaluable for understanding the fragmentation of molecular clouds (e.g. Rosolowsky et al. 2008). We used the dendrogram code developed by Peretto \& Fuller (2009) to extract all clumps from the $\mathrm{NH}_{3}$ derived $\mathrm{H}_{2}$ column density map at regular isocontours. A minimum isocontour value is set at $\mathrm{N}\left(\mathrm{H}_{2}\right)=1 \times 10^{22} \mathrm{~cm}^{-2}$ defining the "trunk" of the dendrogram tree, with a regular isocontour spacings at $1 \sigma$ of $0.04 \times 10^{22} \mathrm{~cm}^{-2}$ throughout, and a $5 \sigma$ detection threshold. A pixel limit of seven defines the smallest size of structure considered resolved, as it roughly matches the beam shape. Cores were identified as the highest levels in the dendrogram hierarchy 
Table 2. Observed and calculated properties of extracted cores.

\begin{tabular}{|c|c|c|c|c|c|c|c|c|c|c|c|c|c|}
\hline ID & $\begin{array}{c}\text { RA } \\
(\mathrm{J} 2000)\end{array}$ & $\begin{array}{c}\text { Dec } \\
(\mathrm{J} 2000)\end{array}$ & $\begin{array}{l}\text { Min. } \\
\left({ }^{\prime \prime}\right)\end{array}$ & $\underset{\left({ }^{\prime \prime}\right)}{\text { Maj. }}$ & $\begin{array}{l}\text { PA } \\
\left({ }^{\circ}\right)\end{array}$ & $\begin{array}{l}\text { Aspect } \\
\text { ratio }\end{array}$ & $\begin{array}{c}\mathrm{N}\left(\mathrm{H}_{2}\right) \\
\left(10^{22} \mathrm{~cm}^{-2}\right)\end{array}$ & $\begin{array}{c}\text { Radius } \\
\left({ }^{\prime \prime}\right)\end{array}$ & $\begin{array}{l}\text { Radius } \\
\text { (pc) }\end{array}$ & $\begin{array}{c}\text { Mass }_{\text {low }} \\
\left(M_{\odot}\right)\end{array}$ & $\begin{array}{c}\text { Mass }_{\text {up }} \\
\left(M_{\odot}\right)\end{array}$ & $\alpha_{\text {vir }}$ & $\begin{array}{l}\text { MM } \\
\text { assoc. }\end{array}$ \\
\hline 1 & $18: 14: 29.5$ & $-17: 32: 14.9$ & 2.3 & 5.0 & 10.3 & 2.2 & 2.22 & 3.0 & 0.07 & 0.5 & 5.4 & 1.47 & - \\
\hline 2 & $18: 14: 24.0$ & $-17: 31: 37.7$ & 2.6 & 9.9 & -32.6 & 3.8 & 2.90 & 4.5 & 0.09 & 1.9 & 14.4 & 0.54 & MM16 \\
\hline 3 & $18: 14: 34.3$ & $-17: 34: 26.9$ & 3.0 & 3.7 & 8.4 & 1.2 & 2.95 & 3.0 & 0.07 & 0.5 & 7.2 & 1.43 & - \\
\hline 4 & $18: 14: 34.7$ & $-17: 34: 06.5$ & 3.5 & 6.5 & -7.2 & 1.8 & 2.94 & 3.7 & 0.08 & 0.6 & 10.3 & 1.49 & - \\
\hline 5 & $18: 14: 33.5$ & $-17: 30: 35.3$ & 2.7 & 5.7 & -35.3 & 2.1 & 3.02 & 3.4 & 0.08 & 0.7 & 9.1 & 2.00 & - \\
\hline 6 & $18: 14: 24.4$ & $-17: 31: 53.3$ & 1.7 & 5.7 & -26.5 & 3.3 & 3.30 & 2.7 & 0.07 & 0.4 & 7.1 & 0.56 & MM17 \\
\hline 7 & $18: 14: 25.2$ & $-17: 32: 02.9$ & 2.9 & 8.8 & -26.8 & 3.0 & 3.37 & 4.4 & 0.09 & 1.2 & 15.8 & 0.54 & MM13 \\
\hline 8 & $18: 14: 26.2$ & $-17: 32: 22.1$ & 2.8 & 6.6 & -25.7 & 2.3 & 3.84 & 4.0 & 0.08 & 1.1 & 15.0 & 0.65 & MM15 \\
\hline 9 & $18: 14: 35.6$ & $-17: 30: 24.5$ & 1.9 & 2.8 & -85.3 & 1.5 & 3.80 & 1.5 & 0.05 & 0.2 & 4.1 & 4.15 & - \\
\hline 10 & $18: 14: 35.6$ & $-17: 30: 54.5$ & 3.7 & 6.8 & 5.3 & 1.8 & 4.22 & 4.6 & 0.10 & 2.6 & 21.8 & 0.61 & MM3 \\
\hline 11 & $18: 14: 35.6$ & $-17: 30: 38.9$ & 1.6 & 3.3 & 17.9 & 2.1 & 3.87 & 1.7 & 0.05 & 0.2 & 4.5 & 1.50 & - \\
\hline 12 & $18: 14: 33.7$ & $-17: 31: 17.3$ & 3.9 & 7.4 & 33.4 & 1.9 & 4.35 & 5.0 & 0.10 & 3.5 & 25.9 & 0.45 & MM5 \\
\hline 13 & $18: 14: 34.6$ & $-17: 30: 46.1$ & 4.7 & 6.9 & 28.1 & 1.5 & 4.64 & 5.3 & 0.11 & 4.2 & 30.8 & 0.64 & - \\
\hline 14 & $18: 14: 33.0$ & $-17: 31: 17.3$ & 1.2 & 4.9 & 34.5 & 4.1 & 4.20 & 1.7 & 0.05 & 0.1 & 4.9 & 0.84 & - \\
\hline 15 & $18: 14: 33.3$ & $-17: 34: 06.5$ & 2.4 & 7.8 & -5.5 & 3.2 & 4.43 & 3.9 & 0.08 & 0.9 & 16.9 & 0.87 & - \\
\hline 16 & $18: 14: 33.1$ & $-17: 34: 19.7$ & 2.2 & 4.4 & -18.6 & 2.0 & 4.35 & 2. & 0.07 & 0.3 & 8.9 & 0.88 & MM7 \\
\hline 17 & $18: 14: 31.2$ & $-17: 33: 07.7$ & 2.7 & 11.9 & 0.7 & 4.4 & 4.59 & 5.2 & 0.11 & 2.2 & 29.6 & 0.43 & - \\
\hline 18 & $18: 14: 27.1$ & $-17: 32: 40.1$ & 2.7 & 7.8 & -32.9 & 2.9 & 4.86 & 4.2 & 0.09 & 2.3 & 20.9 & 0.47 & MM11 \\
\hline 19 & $18: 14: 32.6$ & $-17: 31: 35.3$ & 3.0 & 11.7 & 1.3 & 3.9 & 5.05 & 5.5 & 0.11 & 3.5 & 36.0 & 0.25 & MM9 \\
\hline 20 & $18: 14: 32.3$ & $-17: 32: 01.7$ & 1.1 & 6.5 & 21.2 & 5.9 & 4.73 & 1.8 & 0.06 & 0.1 & 6.0 & 0.73 & - \\
\hline 21 & $18: 14: 29.5$ & $-17: 32: 49.7$ & 1.7 & 6.3 & -4.4 & 3.8 & 4.83 & 2.4 & 0.06 & 0.3 & 9.0 & 0.92 & - \\
\hline 22 & $18: 14: 31.8$ & $-17: 34: 05.3$ & 3.7 & 6.8 & -38.4 & 1.8 & 5.68 & 4.6 & 0.10 & 2.0 & 29.4 & 0.83 & - \\
\hline 23 & $18: 14: 27.7$ & $-17: 32: 55.7$ & 1.6 & 2.3 & -7.1 & 1.5 & 5.44 & 1.0 & 0.05 & 0.1 & 4.2 & 1.05 & - \\
\hline 24 & $18: 14: 32.0$ & $-17: 32: 28.1$ & 2.2 & 4.7 & 10.9 & 2.2 & 5.87 & 2.7 & 0.07 & 1.0 & 12.6 & 0.75 & MM10 \\
\hline 25 & $18: 14: 32.1$ & $-17: 32: 18.5$ & 1.5 & 4.5 & 13.2 & 2.9 & 5.71 & 2.0 & 0.06 & 0.5 & 8.4 & 0.94 & - \\
\hline 26 & $18: 14: 30.4$ & $-17: 33: 56.9$ & 4.6 & 8.6 & -41.9 & 1.9 & 6.48 & 5.4 & 0.11 & 3.5 & 44.3 & 0.85 & MM4 \\
\hline 27 & $18: 14: 28.4$ & $-17: 33: 29.3$ & 8.1 & 12.6 & -7.4 & 1.6 & 9.11 & 9.9 & 0.18 & 36.7 & 195.7 & 0.28 & MM2 \\
\hline 28 & $18: 14: 29.8$ & $-17: 33: 26.9$ & 6.4 & 11.4 & -37.6 & 1.8 & 8.20 & 8.1 & 0.15 & 11.6 & 119.2 & 0.30 & - \\
\hline
\end{tabular}

Notes. Column 1: core ID number; Cols. 2 and 3: right ascension and declination of the core peak emission; Cols. 4 and 5: core major and minor axes; Col. 6; position angle; Col. 7: major-to-minor axes ratio; Col. 8: mean value of $\mathrm{H}_{2}$ column density within core boundary; Col. 9: deconvolved core radius in arcseconds; Col. 10: deconvolved core radius in parsec; Col. 11: lower limit on the mass; Col. 12: upper limit on mass; Col. 13: Virial ratio, calculated using Eq. (7) where the upper limit on the mass was used; Col. 14: Core associations to the MM identifications of previous work (Peretto et al. 2014). The detected proto-stellar sources are MM4, MM5 and MM7. Systematic error from the kinematical distance to SDC13 $(3.6 \pm 0.4 \mathrm{kpc})$ is associated to all size parameters. Uncertainty on the column density and masses originate from the assumption of constant $\left[\mathrm{NH}_{3}\right] /\left[\mathrm{H}_{2}\right] \sim 3 \times 10^{-8}$ abundance (Harju et al. 1993) and temperature (12.7 K) in the cloud.

(termed "leaves") meaning that they contain only themselves and no other sub-structures. All observed core properties are listed in Table 2, such as the central core co-ordinates, major and minor axes, position angle, aspect ratio, radius and mean $\mathrm{H}_{2}$ column density. Core radii were calculated by considering each isocontour core boundary a disc with an equivalent area. The smallest deconvolved core radius can be seen to match half of the beam width, a direct effect of matching the pixel limit of the extraction code to the beam.

We identified 28 cores, of which seven lie along each of the north-west and north-east filaments, three lie along the southeast filament and two along the north filament (plotted in the right panel of Fig. 5). The remaining cores mostly lie at the end of the north-east filament. Their starless (or proto-stellar) nature was identified by the lack (or presence) of Spitzer 8 and $24 \mu \mathrm{m}$ sources. Only three proto-stellar sources were detected, which were already identified by (Peretto et al. 2014; listed in Table 2). Core masses were calculated by taking the mean $\mathrm{H}_{2}$ column density (middle panel of Fig. 5, calculated from the $\mathrm{NH}_{3}$ column density) within the core boundary, and setting the average molecular weight, $\mu=2.8$. Two mass estimates were calculated for each core, one excluding the outer column density shell (effectively excluding the "background", equivalent to the "clipping" scheme of Rosolowsky et al. 2008) regarded as the lower limit of the mass, and one that incorporates the background column density (equivalent to the "bijection" scheme of Rosolowsky et al. 2008), regarded an upper limit of the mass. Small column density peaks on top of a large background column density will generate very different mass estimates. The cores we identify here range from low- to high-mass, with the most massive ones being located near the filament junctions (core IDs 26, 27 and 28 - see Fig. 5). Both mass ranges, as well as the virial ratio (see Sect. 5.5) are listed in Table 2. The association of the newly identified cores with those published in Peretto et al. (2014) is also provided in the table. The dominant source of uncertainties in the estimate of core properties are the distance $(11 \%)$, the abundance (within a factor of two) and the way cores are defined (bijection versus clipping - see Table 2 ). We note that uncertainties related to distance and abundance are systematic and therefore will not change observed trends.

As already mentioned in Sect. 3.1.3, we do observe a correlation between the increase of velocity width and the presence 


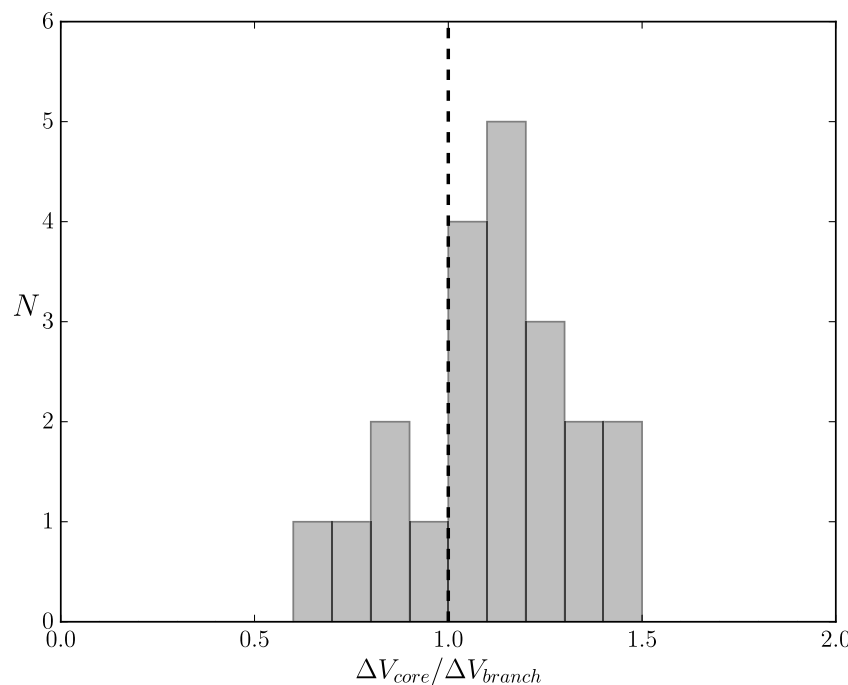

Fig. 6. Histogram of the ratio of the average velocity width within a core region $\left(\Delta V_{\text {core }}\right)$ to that of the underlying branch structure $\left(\Delta V_{\text {branch }}\right)$ in the dendrogram tree. The vertical dashed line indicates where $\Delta V_{\text {core }}=\Delta V_{\text {branch }}$.

of cores. In order to quantify the number of cores displaying such behaviour, we systematically computed the average velocity width within the extracted core area at its position in the dendrogram tree where it is first identified as a leaf $\left(\Delta V_{\text {core }}\right)$. Doing so we find that $73 \%$ of the cores show an increase in their mean velocity width of $\geq 10 \%$ when compared to the velocity width of the underlying "branch" structure of the dendrogram tree $\left(\Delta V_{\text {branch }}\right.$, as shown in Fig. 6). Furthermore, $87.5 \%$ of these belong to the starless core population (according to the lack of mid-infrared point source). To visually demonstrate this increase even further, we split the cores into two sub-samples. The first contains cores with $\Delta V_{\text {core }} / \Delta V_{\text {branch }}>1$, whilst the second contains cores with $\Delta V_{\text {core }} / \Delta V_{\text {branch }} \leq 1$.

In Fig. 7 we stack each of the sub-samples separately and take the mean velocity width (for starless cores only). The overplotted contours are of the average $\mathrm{H}_{2}$ column density. This figure clearly shows that the velocity dispersion is increased over the entire extent of the cores, with a peak towards their centres.

\subsection{Filaments}

The orientation and morphology of all filamentary structures were constrained by identifying the spines of the filaments. As the name suggests, the spine is considered the backbone of a filament, tracing the pixels where the column density exhibits a local maximum in at least one direction. This was quantified by computing the second derivative matrix (e.g. Schisano et al. 2014), that is the Hessian matrix, for each pixel in the column density map. Then, by diagonalising the matrix, and selecting areas where at least one of the eigenvalues is negative, we can identify the filament areas. To reduce this to a spine, we used the THIN IDL function. The local orientation of the filaments at each pixel along the spine is provided by the angle of the first eigenvector and the $x$ axis of the image. Figure 5 shows the spines of each of the four identified filaments, and Table 3 compiles their properties. The masses of all filaments (including the portions of the spines that intersect the central hub) total to $\sim 1000 M_{\odot}$, equal the mass quoted by Peretto et al. (2014). Separating the central hub region from the filaments places half the total mass in the hub region and half in the filaments.

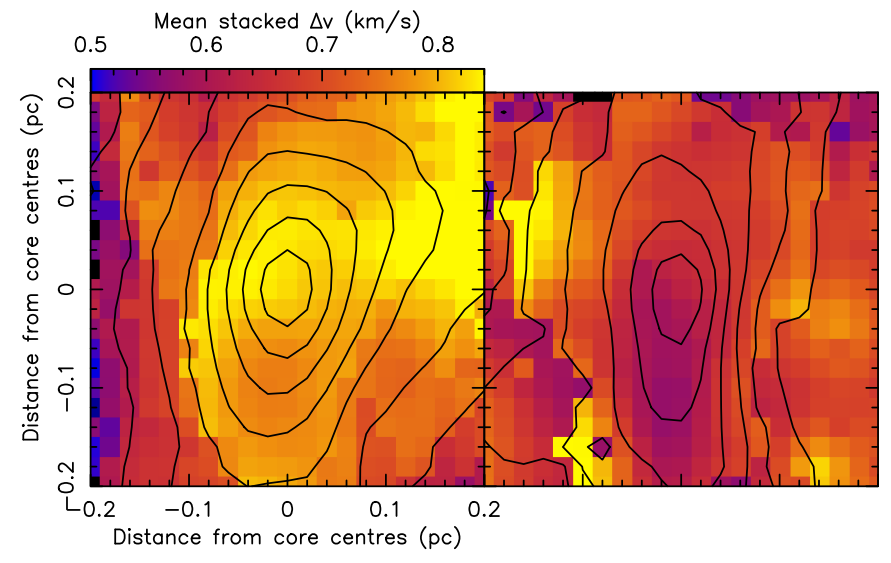

Fig. 7. Average stacked velocity width of cores identified with $\Delta V_{\text {core }} / \Delta V_{\text {branch }}>1$ (left) and those with $\Delta V_{\text {core }} / \Delta V_{\text {branch }}<1$ (right). Included in the construction of the left and right panels are 12 and 5 cores, respectively. Only starless cores within the filamentary arms are included, excluding both proto-stellar and central hub region sources. Contours are of the respective average stacked column density in $0.5 \times 10^{22} \mathrm{~cm}^{-2}$ steps from 0 to $6 \times 10^{22} \mathrm{~cm}^{-2}$ on the left, and in $0.4 \times 10^{22} \mathrm{~cm}^{-2}$ steps from 0.2 to $3.8 \times 10^{22} \mathrm{~cm}^{-2}$ on the right.

\subsubsection{Longitudinal filament profiles}

We first study the filaments along their spines. Figure 8 plots the line profiles of all four filaments in $\mathrm{H}_{2}$ column density (first row), velocity width (second row) and absolute centroid line-of-sight velocity (third row) where the origin of the filaments was defined to reside at the centre of the hub. Filaments show variations in all quantities, and on a range of spatial scales. First, we notice that these variations are correlated in all three quantities, being particularly obvious for the north-east and north-west filaments. More specifically, core positions (marked by shaded regions on all panels) correlate with the velocity width, but also in centroid velocity where local velocity gradients are developed. This is shown in the fourth row of Fig. 8 where we plot the absolute velocity gradient along the spine, evaluated over the mean core size of $0.1 \mathrm{pc}$. We note that a significant fraction of the cores $(\sim 63 \%)$ are located at a peak of velocity gradient. In the bottom row of Fig. 8 we plot the rotational temperature derived from the $\mathrm{NH}_{3}$ emission. Although quite variable along the length of the filament, we do not find a correlation between the temperature and core positions, nor the kinematic properties. We conduct Spearman's rank correlation coefficient test on these correlations for all four filaments separately. We find a definite correlation between the column density and velocity width peaks in every filament (as shown in Fig. 9) with vanishingly small $p$-values in every filament with coefficients ranging from 0.24 in the north-east filament (indicating a moderate correlation), to $0.74-0.91$ in the other three filaments (indicating a strong correlation). We find similarly small $p$-values in the column density and centroid velocity correlations, showing to us a definite link between all three of these properties. Furthermore, we notice that there are other common features to all filaments, such as the strongest column density and velocity dispersion peaks located at the origin of the filaments. Note that this is not true for the north-east filament, but would likely be true if the abundance of ammonia towards the MM1 proto-stellar core (Peretto et al. 2014 labelled in the left panel of Fig. 5) were not decreased (see Sect. 3.1.1). More generally, it is interesting to note that similarities emerge in the large scale morphology of all profiles between pairs of filaments (the north-west and 
Table 3. Summary of filament properties.

\begin{tabular}{|c|c|c|c|c|c|c|c|c|c|c|}
\hline Filament & $\begin{array}{l}\text { Length } \\
\text { (pc) }\end{array}$ & $\begin{array}{c}\text { Orientation } \\
\left({ }^{\circ}\right)\end{array}$ & $\begin{array}{l}\lambda_{\text {core }} \\
(\mathrm{pc})\end{array}$ & $\begin{array}{l}\text { Width } \\
(\mathrm{pc})\end{array}$ & $\begin{array}{c}M_{\text {line }} \\
\left(M_{\odot} \mathrm{pc}^{-1}\right) \\
\end{array}$ & $\begin{array}{c}M_{\mathrm{fil}} \\
\left(M_{\odot}\right)\end{array}$ & $\begin{array}{c}\tau_{\text {crit }} \\
(\mathrm{Myr})\end{array}$ & $\begin{array}{c}\dot{M} \\
\left(M_{\odot} \mathrm{pc}^{-1} \mathrm{Myr}^{-1}\right)\end{array}$ & $\begin{array}{c}\tau_{\text {age }} \\
(\mathrm{Myr}) \\
\end{array}$ & $\begin{array}{c}\left|\nabla V_{r}\right| \\
\left(\mathrm{km} \mathrm{s}^{-1} \mathrm{pc}^{-1}\right)\end{array}$ \\
\hline North (N) & 1.32 & -8.1 & - & 0.27 & 270 & 259 & - & - & - & 0.3 \\
\hline North-West (NW) & 3.06 & -25.6 & $0.34 \pm 0.06$ & 0.16 & 147 & 393 & 0.64 & 48.9 & 3.0 & 0.2 \\
\hline South-East (SE) & 1.17 & +145.2 & $0.41 \pm 0.02$ & 0.37 & 374 & 223 & 0.78 & 40.0 & 9.4 & 1.1 \\
\hline North-East (NE) & 2.89 & +18.0 & $0.33 \pm 0.21$ & 0.25 & 264 & 401 & 0.62 & 50.5 & 5.3 & 1.5 \\
\hline
\end{tabular}

Notes. Column 1: filament name; Col. 2: the length of the filament spine in parsec; Col. 3: the mean orientations from the minimisation of the eigenvalues of the Hessian matrices, in degrees. The origin at $0^{\circ}$ was defined by the north direction, and the positive values are anticlockwise; Col. 4: median core separation, excluding hub cores, quoted with their standard deviation. Given we have excluded hub centre cores, this leaves only one core in the north filament; Col. 5: mean filament width in parsec (from 2.35 times the standard deviation of each radial column density) evaluated from the regions of the filament outside the hub centre only; Col. 6: mass per unit length, evaluated from the integration of radial column density profiles outside the cloud hub; Col. 7: the total mass of the filament, including the regions that pass through the cloud hub centre; Col. 8: the lower limit on the age of the filament (Eq. (1)) (in-evaluable for the north filament given its single core); Col. 9: the accretion rate of material onto the filament during its formation. Col. 10; the time elapsed since the filament became critical; Col. 11: mean radial velocity gradient across the inner $0.2 \mathrm{pc}$ filament width. Systematic errors from the kinematical distance to SDC13 $(3.6 \pm 0.4 \mathrm{kpc})$ and unknown filament inclination are associated to the filament lengths and mean core separations. Inclination angle affects the timescales quoted, as discussed in Sect. 5.2.

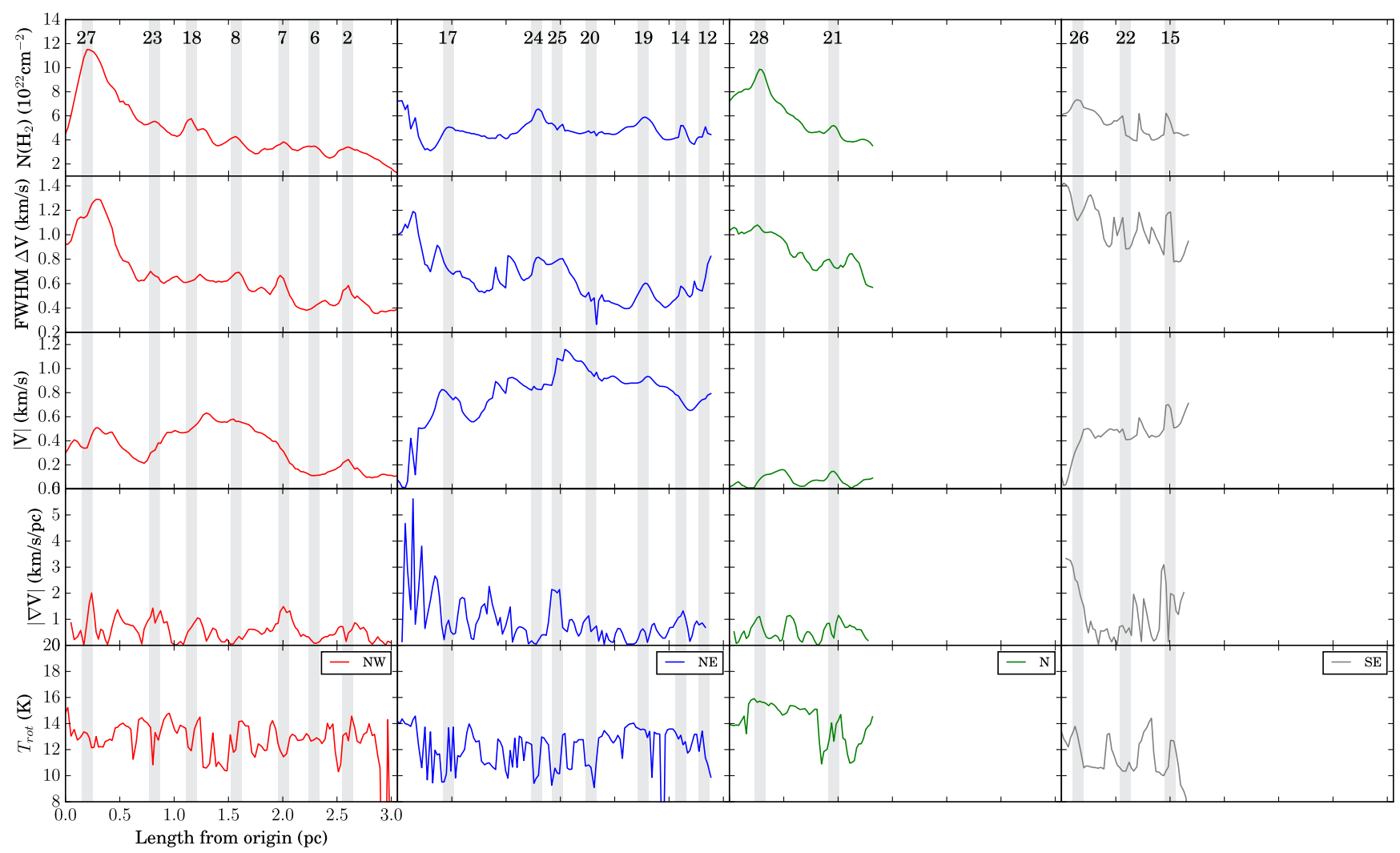

Fig. 8. Profiles along the spines, where each column denotes a different filament (north-west in red, north-east in blue, north in green and southeast in grey). The origin of each spine was centred at the hub region. The first row plots the column density of $\mathrm{H}_{2}$ calculated from the fitted $\mathrm{NH}_{3}$ integrated intensity. The second row plots the FWHM velocity width, while the third row plots the absolute centroid line-of-sight velocity (offset from the cloud systemic velocity of $37 \mathrm{~km} \mathrm{~s}^{-1}$ ), both also from the hyperfine structure fitting. The fourth row plots the absolute velocity gradient evaluated over the mean core size of $\sim 0.1 \mathrm{pc}$. The fifth row plots the rotational temperature derived from the $\mathrm{NH}_{3}$ emission (see Appendix B.2) which has a standard deviation of $1.8 \mathrm{~K}$. The drops of temperature below $8 \mathrm{~K}$ are artificial and due to missing $\mathrm{NH}_{3}(2,2)$ detections at these particular locations. The vertical shaded regions in each panel correspond to the peak $\mathrm{N}\left(\mathrm{H}_{2}\right)$ positions of the cores along the spine, and are $\sim 0.1 \mathrm{pc}$ wide.

north-east filaments on one side, and the north and south-east filaments on the other). Overall, such similarities are suggestive of the common physical origin of the SDC13 filament evolution, as discussed in detail throughout Sect. 5.

\subsubsection{Radial filament profiles}

To construct a radial view of the filaments, we interpolate along an $0.4 \mathrm{pc}$ slice perpendicularly orientated to each spinal pixel using a Taylor expansion method (where the interpolation step is equivalent to half a pixel). Every slice is used to construct radial position-velocity (PV) profiles from the $\mathrm{NH}_{3}$ hyperfine structure fitted cube. Any prevailing velocity gradient orientated parallel to the filaments however causes each radial PV slice to be centred differently in velocity, effectively smearing the mean radial PV profile. This was corrected by aligning each PV slice to the central velocity of the slice. 


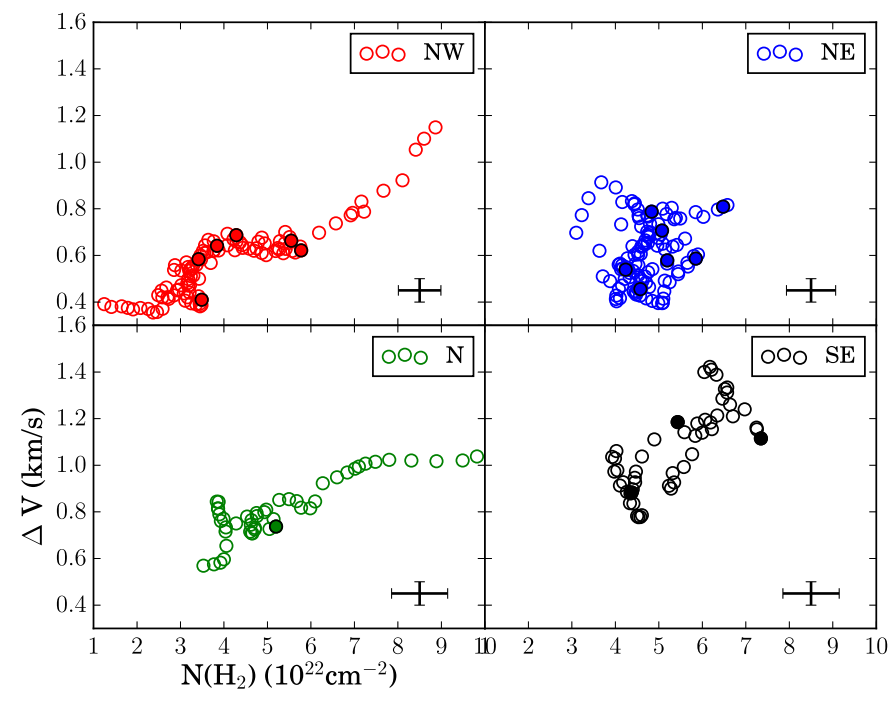

Fig. 9. Correlations of velocity width to $\mathrm{H}_{2}$ column density along the filament spine directions for the north-west (top left) north-east (top right), north (bottom left) and south-east (bottom right) filaments, excluding the portion of the spines that intersect the central hub regions (identified as where the filament can no longer be distinguished from the hub). Filled circles denote the spinal pixel position of the identified cores along the filaments. A mean error is plotted in the bottom right of each panel, equal to $\pm 20 \%$ on the mean $\mathrm{H}_{2}$ column density, and $\pm 0.05 \mathrm{~km} \mathrm{~s}^{-1}$ on $\Delta V$.

It is clear from the on-axis concentric nature of the highest intensity emission in the north and north-west filaments in Fig. 10 that any radial velocity gradient does not dominate the velocity field. The intensity-weighted mean of these profiles does appear flat at their centremost regions around the spinal pixel, with relatively low radial velocity gradients of $0.3 \mathrm{~km} \mathrm{~s}^{-1} \mathrm{pc}^{-1}$ and $0.2 \mathrm{~km} \mathrm{~s}^{-1} \mathrm{pc}^{-1}$, respectively. In contrast, the south-east and north-east filaments have rather significant mean radial velocity components across their entire width, corresponding to 1.1 and $1.5 \mathrm{~km} \mathrm{~s}^{-1} \mathrm{pc}^{-1}$, respectively. Although significant in this context, these gradients are not as strong as others observed by Fernández-López et al. (2014) or Beuther et al. (2015) for example, who observe radial gradients an order of magnitude larger than longitudinal velocity gradients in Serpens South and IRDC 18223, respectively.

The radial variations of the velocity width are plotted in the bottom panels of Fig. 10. These profiles show that the longitudinally averaged velocity dispersion varies across all filaments, with, in some cases, local minima along the filament spine, and local maxima at the edges. However, as discussed in Sect. 4, these trends result from the average of a number of different processes that are mixed-up together in such profiles.

\subsection{JVLA/GBT combined versus JVLA-only datasets}

In previous sections, results obtained from the combined JVLA/GBT dataset were presented. As already mentioned in Sect. 2.3, such a combined dataset is optimal to recover emission at all spatial scales. However, when trying to characterise the gas properties of the densest parts of the filaments (i.e. cores and filament spines) as we have done above, results can be affected by the contamination of the larger scale, more diffuse, foreground/background emission. The effect of this contamination would be to dilute the observational signatures one is trying to identify.

For that reason we redid some of the analysis presented in earlier sections using the JVLA-only dataset, which traces

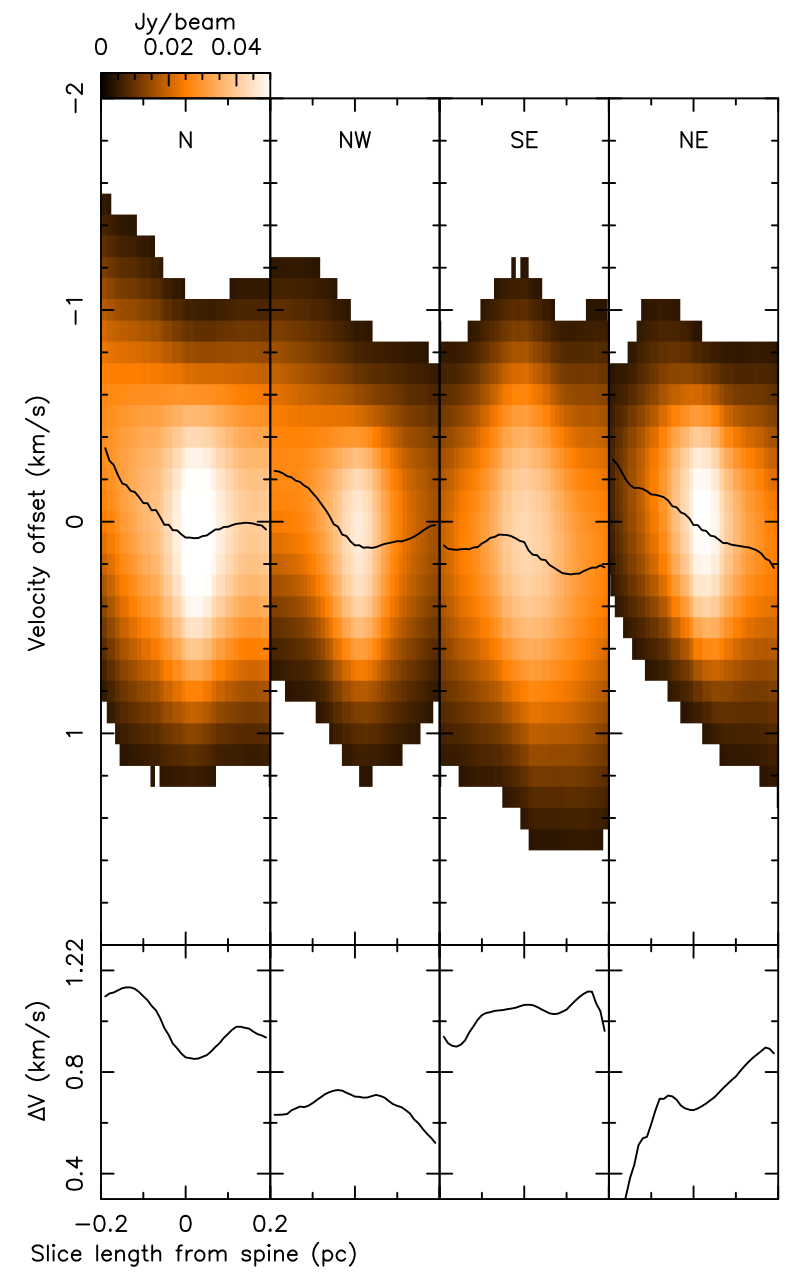

Fig. 10. Top: mean position-velocity diagrams across the north, north-west, south-east and north-east filaments (from left to right), where position denotes the length of a perpendicular slice relative to the central spine pixel in parsec, and the velocity are relative to the peak velocity of each slice. The overplotted black line shows the trend of the intensity-weighted mean offset velocity at each position along the radial slice. Mean radial gradients were computed within the inner $0.2 \mathrm{pc}$ width around the spinal pixel. Bottom: mean variation in the velocity width in the radial direction, in $\mathrm{km} \mathrm{s}^{-1}$.

only the most compact/densest regions of the cloud. We particularly focus on the velocity dispersion variations along the filament spines and towards the cores. By fitting the hyperfine components of the JVLA-only $\mathrm{NH}_{3}$ emission, we find that the increase of the velocity width at the core positions is even larger than in the combined data set. This is illustrated in Figs. 11 and 12, reproductions of Figs. 7 and 8 but of the JVLAonly data. From these figures, one can see that the JVLA-only velocity width towards the core centres is 1.5-2 times larger than that of the surroundings, compared to a 1.1-1.4 factor in the JVLA/GBT combined data. We also notice that in the JVLAonly data the remaining starless cores also display an increase of velocity width. This analysis strengthens our conclusions about the local increase of velocity dispersion towards the SDC13 starless cores.

\section{Discussion}

In this section, we discuss the various observational properties of the filaments in SDC13 in terms of filament evolution theories. 
(a) JVLA+GBT combined
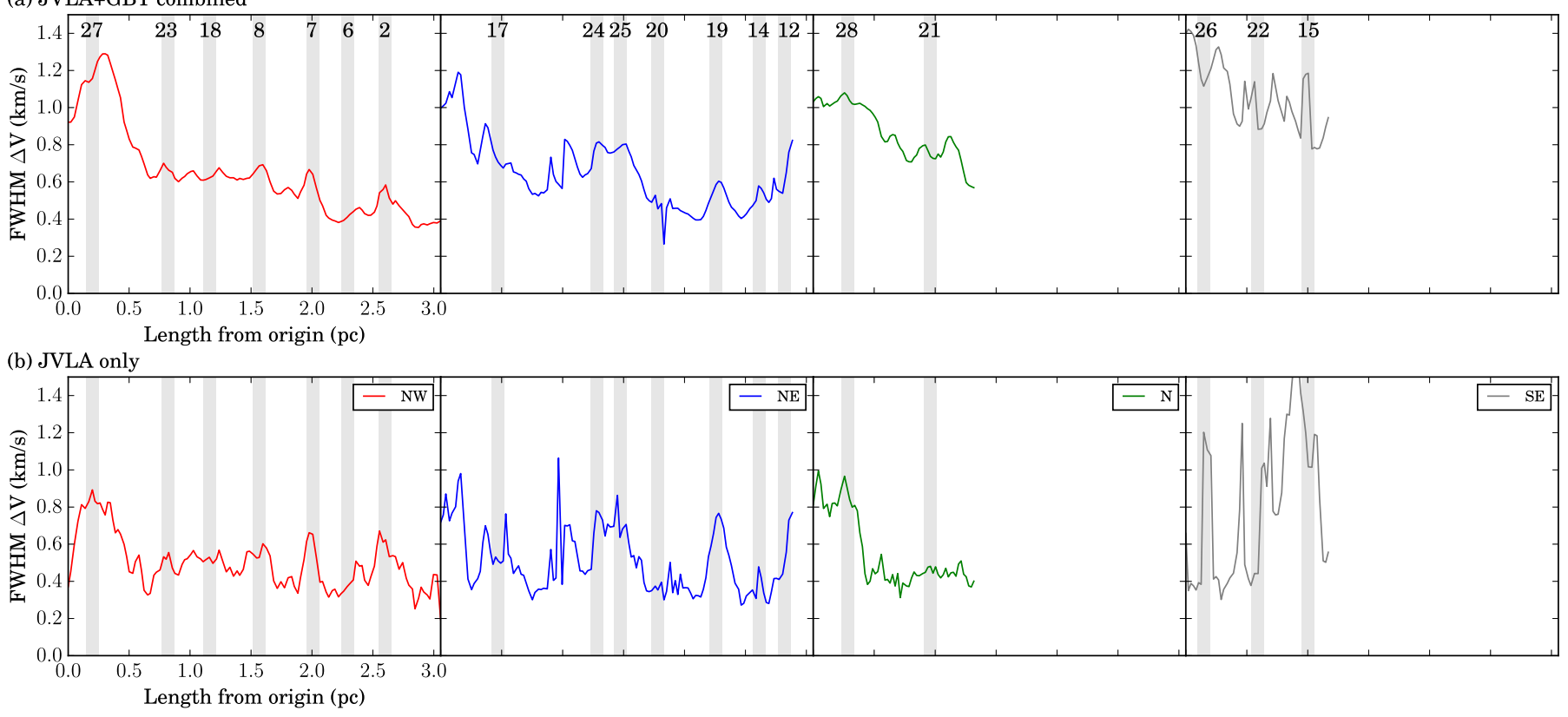

Fig. 11. Velocity width profiles of the north-west (red), north-east (blue), north (green) and south-east (grey) filaments in the combined data (top row, as presented in the second row of Fig. 8) and the JVLA-only data (bottom row), evaluated over the same filament spines as identified in Sect. 4.2.

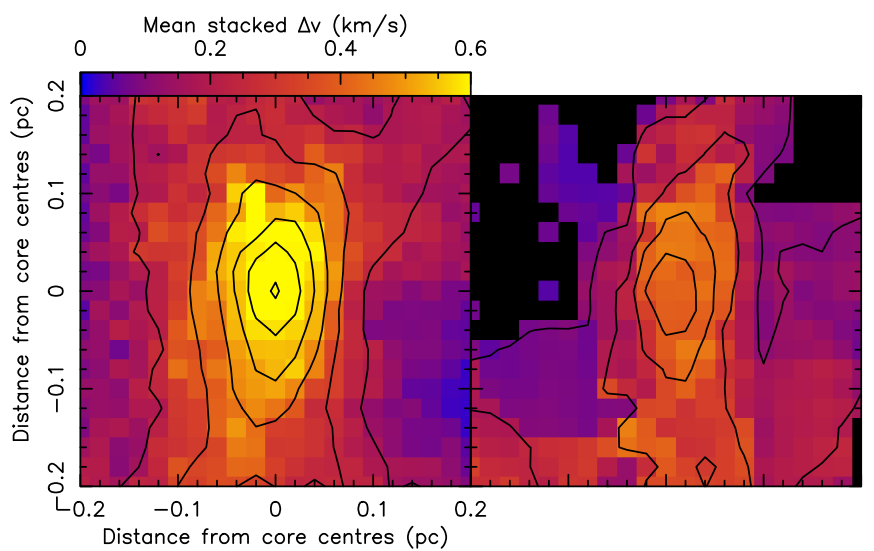

Fig. 12. Reproduction of Fig. 7, but for the JVLA-only data. The average stacked velocity width of cores identified with velocity width peaks (left) and those without peaks (right). Included in the construction of the left and right panels are 13 and 4 cores, respectively. Plot details are the same as those detailed in the caption of Fig. 7.

To facilitate the visualisation of the filament properties, the radial interpolation carried out in Sect. 4.2.2 was carried out on the column density, centroid velocity, velocity width and Spitzer $8 \mu \mathrm{m}$ opacity for all filaments. Aligning each slice to the pixel that lies along the filament spines gives us a unique deprojected perspective of the filaments (Fig. 13) i.e. a view of the filament independent of their on-sky projection, making some of the filament features discussed below stand out.

\subsection{Supercritical filaments}

One important parameter of interstellar filaments regarding their stability is their mass-per-unit-length, $M_{\text {line }}$. The first step towards measuring this quantity is to determine their radial column density profiles. In our attempt to compute these profiles, neither a Gaussian distribution nor a Plummer profile (Whitworth \& Ward-Thompson 2001) accurately reflects the complexity of the radial column density distributions (unlike Arzoumanian et al. 2011, for example, see Appendix E). Therefore, we instead integrate all radial column density distributions using the Trapezoidal rule at every position along the filaments spines to calculate the mass per unit length. A rough estimation of the filament widths were obtained by taking $\sqrt{8 \ln 2}$ times the standard deviation of the radial column density profiles. In calculating the mean $M_{\text {line }}$ and filament widths (listed in Table 3), we excluded the regions that intersected the cloud hub (identified as belonging to the radial column density slices where the extended nature of the hub emission makes the determination of the width impractical). Figure 14 plots the evolution of the filament width and $M_{\text {line }}$ along the filament lengths, respectively.

The critical mass per unit length, $M_{\text {line,crit }}=2 a_{0}^{2} / G$ (where $a_{0}$ is the isothermal sound speed and $G$ is the gravitational constant) is the critical value above which an interstellar filament becomes gravitationally unstable to radial contraction and fragmentation (Ostriker 1964). For a typical $10 \mathrm{~K}$ filament, $M_{\text {line,crit }}=16 M_{\odot} \mathrm{pc}^{-1}\left[\frac{T}{10 K}\right]$. At the mean rotational temperature of SDC13 of $12.7 \mathrm{~K}, a_{0}=0.21 \mathrm{~km} \mathrm{~s}^{-1}$ and the critical mass per unit length is $20.4 M_{\odot} \mathrm{pc}^{-1}$. All SDC13 filaments can be classed as thermally supercritical (see Table 3) therefore prone to radial gravitational contraction and fragmentation along their lengths. When considering the effective sound speed, $a_{\mathrm{eff}, 0}=\sqrt{a_{o}^{2}+\sigma_{\mathrm{NT}, 0}^{2}}=0.26 \pm 0.02 \mathrm{~km} \mathrm{~s}^{-1}$ (where $\sigma_{\mathrm{NT}, 0}=0.15 \pm 0.02 \mathrm{~km} \mathrm{~s}^{-1}$, taken from Fig. 17 as the most representative value of the filament dense gas prior to fragmentation - see Sect. 5.4) the critical mass per unit length becomes $31.3 M_{\odot} \mathrm{pc}^{-1}$, a factor of 1.5 larger than the previously calculated thermal $M_{\text {line,crit }}$, but still a factor of 4-10 lower than the measured filament line masses.

\subsection{Core separation and age estimates}

The separation of cores along filaments can provide indication on the physical mechanism driving the fragmentation. The top 


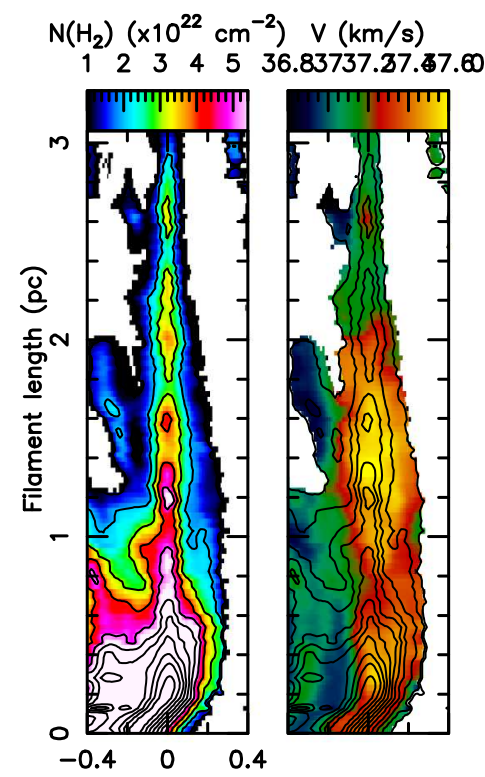

$\Delta V(\mathrm{~km} / \mathrm{s}) \quad 8 \mu \mathrm{m}$ opacity

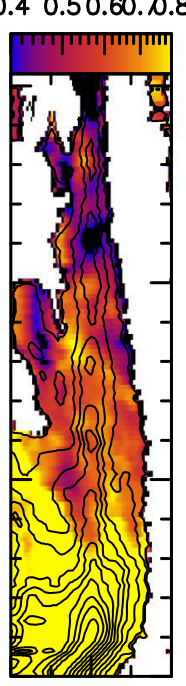

Slice length from spine $(p c)$

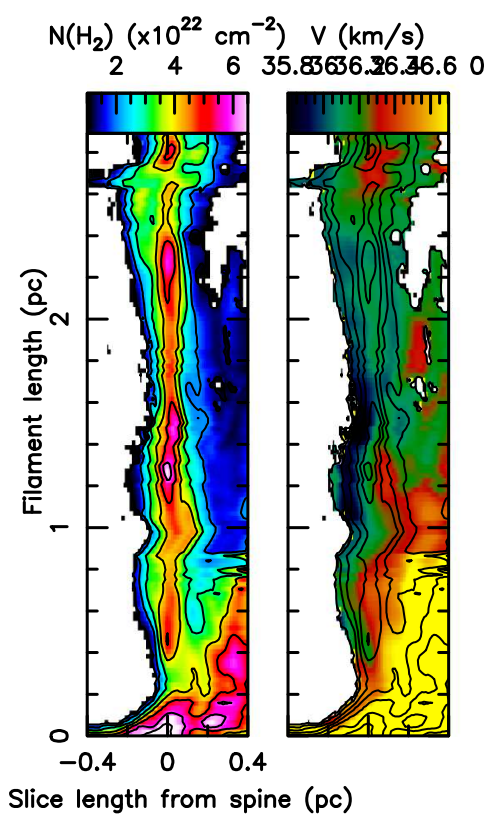

$\Delta V(\mathrm{~km} / \mathrm{s}) \quad 8 \mu \mathrm{m}$ opacity

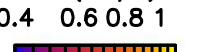

0.511 .52
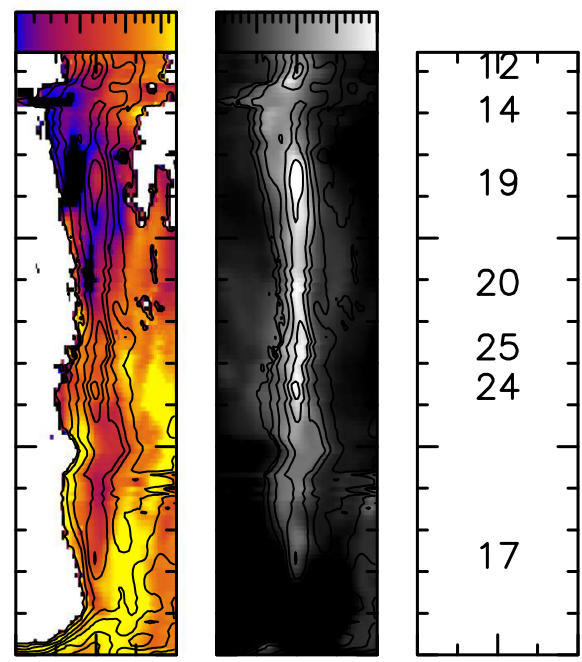

Fig. 13. Deprojected views of the north-west (top) and north-east (bottom) filaments. The filament length is plotted on the $y$-axis, while the length of the radial slices from the central spine pixel in plotted on the $x$-axis. In both sub-figures, the panels show (from left to right) the $\mathrm{H}_{2}$ column density derived from the $\mathrm{NH}_{3}$ emission, the lineof-sight velocity, the velocity width, the opacity derived from the $8 \mu \mathrm{m}$ Spitzer emission, and finally the core ID as listed in Table 2. Contours in the first four panels are of the column density, from $1 \times 10^{22} \mathrm{~cm}^{-2}$ to $11 \times 10^{22} \mathrm{~cm}^{-2}$, spaced by $1 \times 10^{22} \mathrm{~cm}^{-2}$. The plots for the south-east and north filaments are in Appendix C. panel of Fig. 15 shows the Gaussian Kernel Density Estimation (KDE) plot of core separations in all filaments (excluding hub cores) where the two plotted distributions each use a different bandwidth Gaussian. The core separations were calculated on the original on-sky projected filament spines, and not from the deprojected plots in Fig. 13, although both provide identical results. There is clearly a peak in each distribution at a core separation $\lambda_{\text {core }} \simeq 0.37 \pm 0.16 \mathrm{pc}$. Regular spacings in IRDCs with core separation ranging between $\sim 0.2$ and $0.4 \mathrm{pc}$ have already been reported (Beuther et al. 2015; Henshaw et al. 2016; Zhang et al. 2009). For comparison, we simulated the four filaments by randomly placing the same number of cores as observed along each of the four filament lengths and calculated the separation of each consecutive core pair (e.g. Teixeira et al. 2016), and repeated the process 100000 times. In doing this, we stipulated that the smallest core separation in these random core distributions must exceed the beam size, equivalent to $0.07 \mathrm{pc}$ spatial scale. We can clearly see that the observed cumulative histogram (black) is steeper than the simulated cumulative histogram (red) in the bottom panel of Fig. 15. To quantify these differences, we used the Kolmogorov-Smirnov test to estimate the probability that these two histograms originate from the same parent distribution. We find that there is a $15 \%$ chance that this is the case, exceeding the significant test limit of 5\%. However, coupled with the relatively large K-S statistic of 0.28 (suggesting a large maximum distance between the two cumulative distributions), we reject the null hypothesis and consider that these two histograms are significantly different, demonstrating that the core spacing in the SDC13 filaments are not randomly distributed. The median value of $\lambda_{\text {core }}$ in each filament is given in Table 3. Note that looking at the deprojected view of the filaments in Fig. 13, the spacing of the north-west filament seems to be more regular than the others. This is supported by the lower core spacing standard deviation of the north-west filament, i.e. $0.06 \mathrm{pc}$ (whilst excluding core number 27 in the hub centre) versus $0.21 \mathrm{pc}$ for the north-east filament.

Inutsuka \& Miyama (1992) and Miyama et al. (1994) showed that filaments in hydrostatic equilibrium fragment under density perturbations whose wavelengths are four times the filament diameter. Looking at the relevant values given in Table 3 we see 


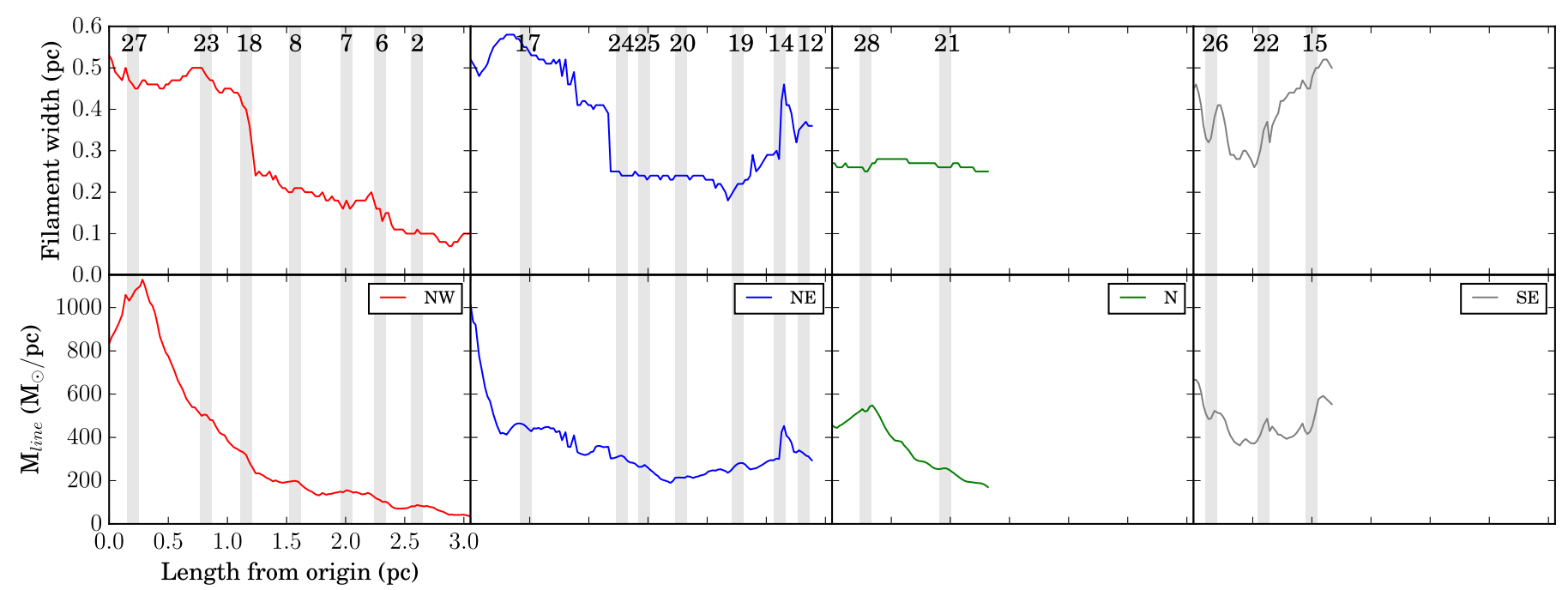

Fig. 14. Caption as for Fig. 8. First row: filament width calculated from 2.35 times the standard deviation of the radial column density distributions. The jump in width seen at roughly $1.2 \mathrm{pc}$ in the north-west and north-east filaments is due to the exit of the filament spine from the hub. The mean filament width was evaluated beyond this point. Second row: mass per unit length, evaluated from conducting a Trapezoidal integration under all radial column density distributions.

that the ratio between the core separation of the SDC13 filaments and their widths varies between 1.1 and 2.1 which, given the unknown inclination, is compatible with a ratio of 4 . However, in a turbulent medium, filaments in equilibrium are likely to be rare objects. Interestingly, Clarke et al. (2016, 2017) studied the fragmentation of non-equilibrium, accreting filaments and showed that the fastest growing mode of density perturbations in such systems, $\lambda_{\text {core }}$, is a function of the time it takes to build-up a critical filament through accretion, $\tau_{\text {crit }}$, and the effective sound speed, $a_{\text {eff, } 0}$ :

$\lambda_{\text {core }}=2 a_{\text {eff }, 0} \tau_{\text {crit }}$.

By measuring the core separation and the gas temperature, it then becomes possible to derive $\tau_{\text {crit }}$. One can also relate $\tau_{\text {crit }}$ to the accretion rate onto the filament $\dot{M}$ by:

$\tau_{\text {crit }}=\frac{M_{\text {line,crit }}}{\dot{M}}$

where $M_{\text {line,crit }}$ is the critical mass per unit length. Values for $\tau_{\text {crit }}$ and $\dot{M}$ (derived using the previously calculated $a_{\mathrm{eff}, 0}=0.26 \mathrm{~km} \mathrm{~s}^{-1}$ and $M_{\text {line,crit }}=31.3 M_{\odot} \mathrm{pc}^{-1}$ ) are given in Table 3 for each filament. According to this model, we see that it takes on average $\sim 0.68 \mathrm{Myr}$ to form the SDC13 filaments up to the critical mass per unit length, with an average accretion rate $\sim 46.5 M_{\odot} \mathrm{pc}^{-1} \mathrm{Myr}^{-1}$. Interestingly, assuming that the derived accretion rate remained constant over the entire filament lifetime, one can derive the age, $\tau_{\text {age }}$, through:

$\tau_{\text {age }}=\frac{M_{\text {line }}}{\dot{M}}$

As shown in Table 3 we obtain an average value of $\tau_{\text {age }} \sim 5.9 \mathrm{Myr}$, which implies that the elapsed time since the filaments became critical is $\sim 5.2 \mathrm{Myr}$. Based on the observed longitudinal velocity gradients and assuming that the gas is freefalling, Peretto et al. (2014) estimated that the SDC13 filaments had been collapsing for $\sim 1-4 \mathrm{Myr}$. This is consistent with the estimate based on the Clarke et al. $(2016,2017)$ model. However, in the calculation of $\tau_{\text {crit }}$ we have not taken into account projection effects in the estimate of $\lambda_{\text {core }}$. Doing so will increase $\tau_{\text {crit }}$ by a factor $1 / \cos (\theta)$ where $\theta$ is the angle between the direction of the main axis of the filament and the plane of the sky, which for an average angle of $67^{\circ}$ (Peretto et al. 2014) is equal to $1.7 \mathrm{Myr}$. This brings the estimated age of the filament to $15.1 \mathrm{Myr}$, more than a factor of 3 larger than the dynamical timescale estimated by Peretto et al. (2014).

\subsection{Collapse timescales}

With all the hierarchical structure in SDC13 extracted using the dendrogram method, we calculate their radii, aspect ratios and mean densities. As the free-fall collapse time of a structure depends only on the density, we calculate $t_{\mathrm{ff}}$ for all extracted structures, from the base of the dendrogram tree up to the leaves using:

$t_{\mathrm{ff}}=\left(\frac{3 \pi}{32 G \rho}\right)^{1 / 2}$,

where $G$ is the gravitational constant, and $\rho$ is the density of the core (for which we use the upper limit on the mass in Col. 12 of Table 2). However, this is not an appropriate approach for nonspherical objects like the high-aspect ratio filamentary structures within SDC13 (Pon et al. 2012; Toalá et al. 2012). Clarke \& Whitworth (2015) derive a collapse timescale $\left(t_{\text {col }}\right)$ valid for both filamentary and near spherical structures:

$t_{\mathrm{col}}=\left(0.49+0.26 A_{\mathrm{o}}\right)(G \rho)^{-1 / 2}$,

where $A_{\mathrm{o}}$ is the aspect ratio, valid down to values of $A_{\mathrm{o}} \gtrsim 2$. The filamentary nature of SDC13 from large to small scale (i.e. $61 \%$ of cores having $A_{\mathrm{o}}>2$ - Table 2) justifies the need to use Eq. (5). Figure 16 shows the collapse time $t_{\text {col }}$ for all identified structures in the SDC13 dendrogram. Overall we see a decrease of the collapse time, from $\sim 0.7$ to $0.1 \mathrm{Myr}$ as we go from large to small structures as a result of the structures' decreasing aspect ratios and increasing densities. A consequence of this hierarchical collapse time is that cores will collapse well before the filaments. This is the basic idea behind the hierarchical star formation models of Vázquez-Semadeni et al. (2009, 2017). On the same figure, 

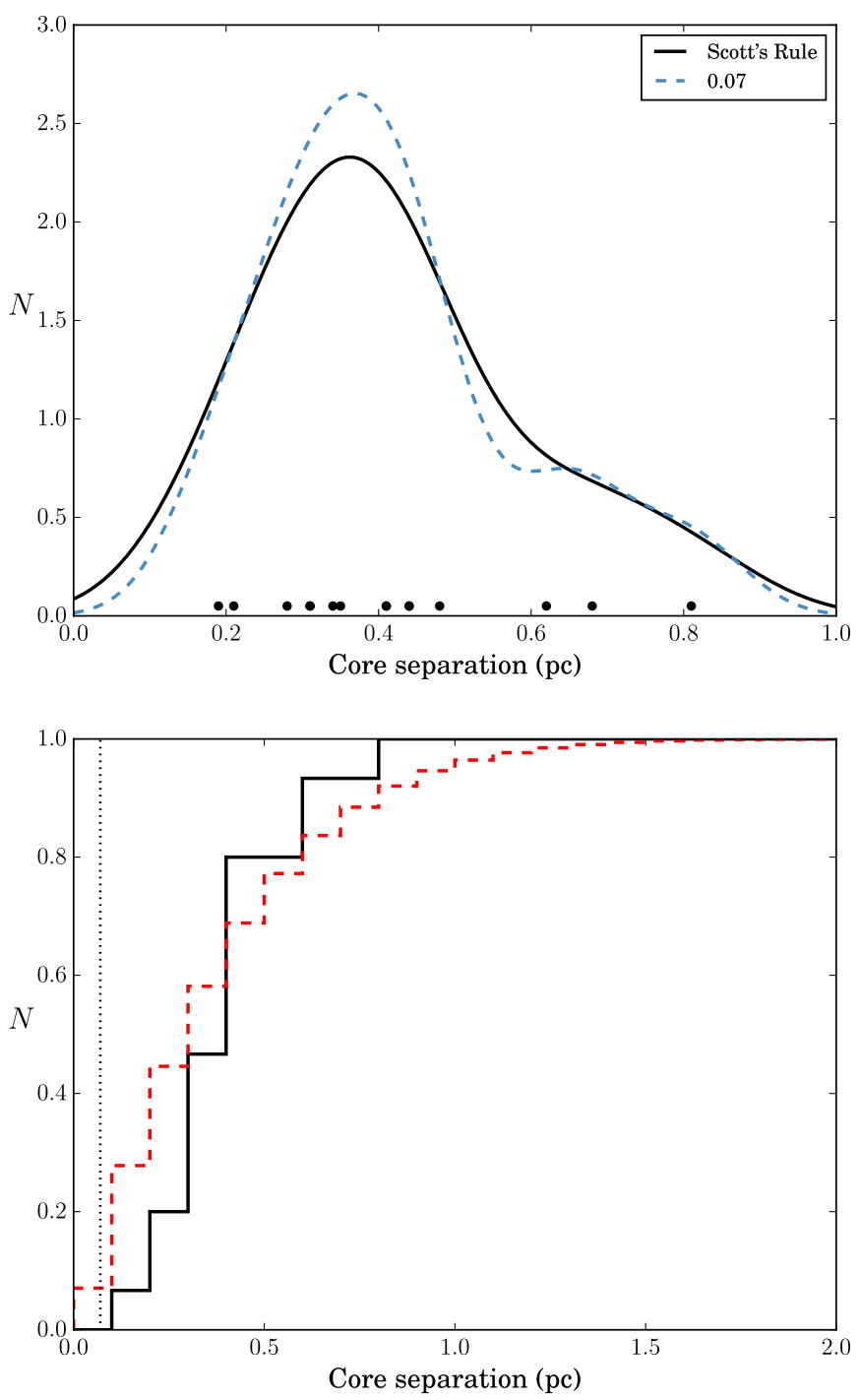

Fig. 15. Top: Gaussian Kernel Density Estimation (KDE) plot of the separation of each consecutive core pair in each of the four filaments. Each line denotes a different bandwidth Gaussian, $0.07 \mathrm{pc}$ (equivalent to the beam size, in dashed blue) and $0.1 \mathrm{pc}$ (derived by the standard Scott's Rule, and equivalent to the mean core radius, in black). Black circles at $N=0.05$ are overplotted to show the positions of the individua spacings in the sample. The standard deviation is $0.16 \mathrm{pc}$. Bottom: normalised, cumulative distribution of the observed core separation (black line) and the 100000 simulated core separations (dashed red line). The bin size in both plots was set to be slightly larger than the beam at $0.1 \mathrm{pc}$ in size. The vertical dotted line in both denotes the data spatial resolution of $0.07 \mathrm{pc}$, used as a cut-off of the allowed simulated spacings.

we see one structure in the north-east filament that departs from the rest of the SDC13 structures, with a longer collapse time than the structure in which it is embedded in. This is due to the particularly long aspect ratio of that particular structure. We also notice that the same structure exhibits the strongest radial velocity gradient (see Sect. 5.2) and the smallest core separation (see Table 3). As speculated later in the paper, we argue that this is a direct consequence of the compression of the pre-existing filament by the feedback of a nearby star formation event.

\subsection{Linewidth as a probe of infall}

The increase of velocity width within the cores can be quantitatively compared to the velocity dispersion within the filaments.
In that respect, we calculated the non-thermal contribution of each pixel within SDC13 following (Myers 1983):

$\sigma_{\mathrm{NT}}=\sqrt{\frac{\Delta V_{\mathrm{obs}}^{2}}{8 \ln 2}-\frac{k_{b} T}{m_{\mathrm{NH}_{3}}}}$

where $\Delta V_{\text {obs }}$ is the fitted FWHM velocity width, $k_{b}$ is the Boltzmann constant, and $m_{\mathrm{NH}_{3}}$ is the mass of an $\mathrm{NH}_{3}$ molecule. To determine whether the filaments are subsonic or supersonic in nature, we compare this non-thermal sigma velocity dispersion to the isothermal sound speed $a_{0}$ at $12.7 \mathrm{~K}$ of $0.21 \mathrm{~km} \mathrm{~s}^{-1}$. Figure 17 shows the histograms of $\sigma_{\mathrm{NT}} / a_{0}$ for all four filaments, where pixels within core regions only are overplotted in white. Overplotted in black are the corresponding distributions taken from the JVLA-only data. We see that in all filaments the gas in the combined data is predominantly supersonic, however, the north-west and north-east filaments also peaks significantly in the sub- and/or transonic regime. This second peak is coincident with that of the JVLA data which only exhibits a minor supersonic tail in its distribution whilst peaking sub- and/or transonically.

Barranco \& Goodman (1998) and Goodman et al. (1998) find that $\sigma_{\mathrm{NT}}$ remains constant across core regions, and increases once outside the core boundary. They call this behaviour velocity "coherence", suggesting that cores have sizes that are intimately linked to the turbulence properties. We observe a different behaviour, with $88 \%$ of pixels within core regions have $\sigma_{\mathrm{NT}} / a_{0} \geq 1$. This is consistent with our systematic identification of $73 \%$ of cores exhibiting a peak in velocity width. However, $87 \%$ of pixels within the surrounding filament also have $\sigma_{\mathrm{NT}} / a_{0} \geq 1$. Despite this similarity in the core and filament pixel distributions, conducting a Kolmogorov-Smirnov test of the two show vanishingly small $p$-values with moderate coefficient values (between 0.2 and 0.3 ), indicating they are significantly different. In the JVLA data however, 55\% of pixels within core regions peak supersonically compared to only $36 \%$ of filament pixels. This further demonstrates that with the addition of the extended background emission in the combined data, the kinematics of the cores have become diluted and similar to that of the surrounding filament. Probing the densest gas with the JVLA-only shows that the kinematics of the cores are more distinct from the surrounding filament.

A decrease of velocity dispersion towards low-mass prestellar cores has been previously observed (Fuller \& Myers 1992; Goodman et al. 1998; Caselli et al. 2002; Pineda et al. 2010, 2015). More recently, this transition to coherence has also been observed towards entire, parsec-long filaments (Hacar \& Tafalla 2011; Hacar et al. 2016). Transonic cores and filaments are expected to form in a turbulent ISM where supersonic shocks generate stagnation regions where turbulent energy has been dissipated (Padoan et al. 2001; Klessen et al. 2005; Federrath et al. 2016). In SDC13, with mostly transonic velocity dispersion (where both the combined and JVLA data sets peak in Fig. 17, hence best representing the dense gas of the system), everything indicates that the filaments represent such post-shock regions, the increase of the dispersion in some localised region at the edge of the filament being reminiscent of what Klessen et al. (2005) sees in their turbulent simulation of core formation. In this context, the velocity dispersion increase towards the core would be then purely generated by gravity (e.g. Ballesteros-Paredes et al. 2017).

Another possible explanation for a local increase of velocity dispersion towards the cores could be the presence of embedded proto-stellar sources. Even though we do not see any 


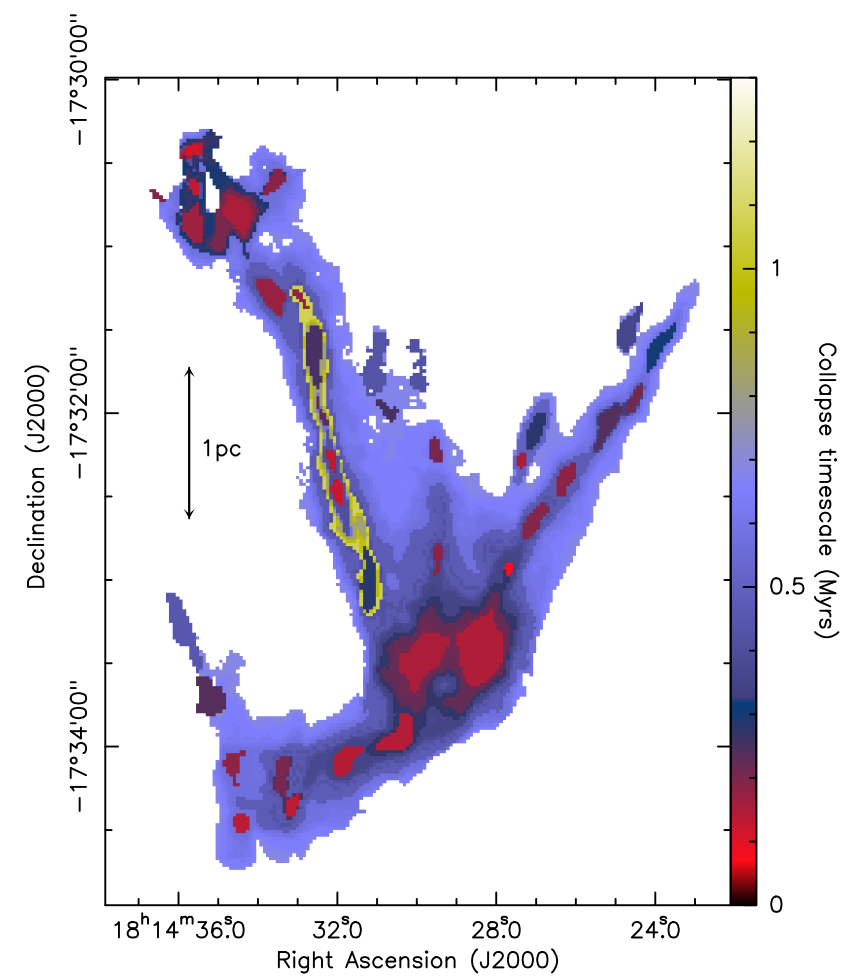

Fig. 16. Map where the outline of all extracted structures are coloured by their collapse timescale $t_{\mathrm{col}}$ in Myr.

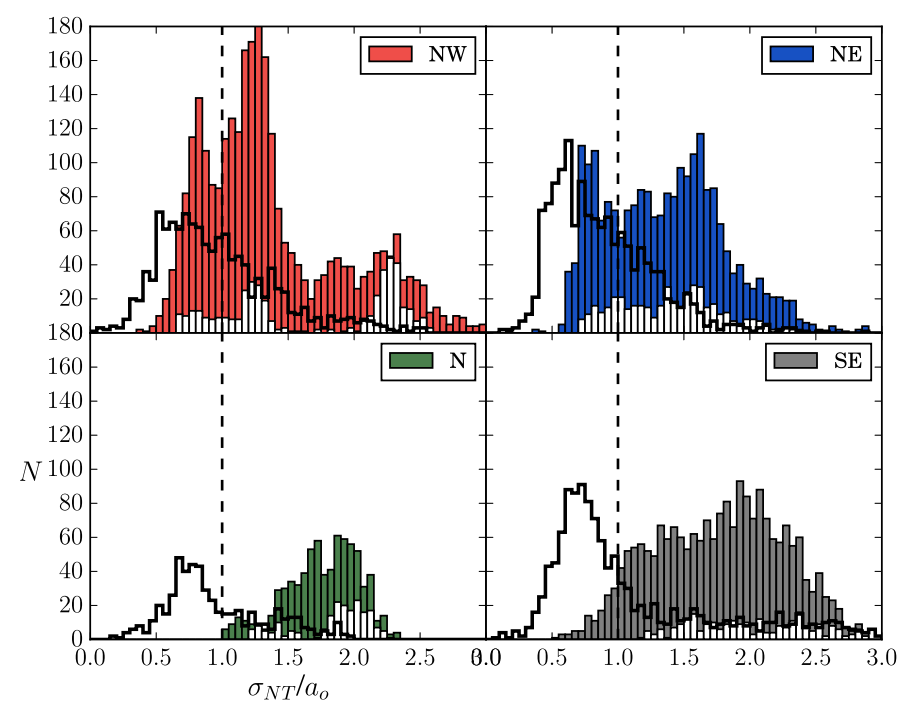

Fig. 17. Non-thermal contribution to the velocity dispersions, divided by the isothermal sound speed at the temperature of the $\mathrm{NH}_{3}$ gas of $12.7 \mathrm{~K}$ i.e. the Mach number. The red histogram is the north-west filament, blue is the north-east, green is the north while grey is the south-east. The vertical dashed line denotes the transition from subsonic to supersonic motions. Overplotted in white in each panel is the Mach number of the core regions only from the combined data. The overplotted black line is the distribution of the JVLA-only velocity dispersion results.

mid-infrared sources towards them, such protostars could be embedded enough to remain undetectable with both Spitzer and Herschel. These protostars could have associated outflows which disrupt the surrounding gas, contributing to a local increase in the velocity dispersion (Duarte-Cabral et al. 2012). ALMA observations of SDC13 cores at sub-arcsecond resolution could settle this issue.

\subsection{Evolution of the virial ratio from large to small scale}

Using the dendrogram tree, we can estimate the virial ratio for all extracted structures. Following Bertoldi \& McKee (1992), the virial ratio of a uniform density sphere is given by:

$\alpha_{\mathrm{vir}}=\frac{5 a_{\mathrm{eff}}^{2} R}{G M}$,

where $R$ is the radius and $M$ is the mass of the cores found by the extraction code, respectively, and $a_{\mathrm{eff}}$ is the total velocity dispersion considering both thermal and non-thermal contributions from all molecules. We use the upper limit on the mass as it matches the amount of gas responsible for the observed velocity dispersion. In the absence of significant magnetic energy density, structures with $\alpha_{\text {vir }}<2$ are thought to be gravitationally bound, those with $\alpha_{\text {vir }} \sim 1$ are compatible with hydrostatic equilibrium, those with $\alpha_{\text {vir }}<1$ are likely to be gravitationally unstable, while structures with $\alpha_{\text {vir }}>2$ are unbound and either dispersing or held together by external pressure. Figure 18 shows the map of virial ratios for all SDC13 structures. Based on this map, it is clear that as we go from large to small scale, the virial ratio increases from $\alpha_{\text {vir }} \sim 0.1$ towards 1 . Such low-values suggest that SDC13 is gravitationally unstable on all scales, and that the transfer of gravitational to kinetic energy could be the process responsible for the increase of the virial ratios on small scales.

Such an evolution of the viral ratio can be analytically described by the following equation:

$\alpha_{\mathrm{vir}}=2 \frac{E_{k, 0}}{\left|E_{g}\right|}+2 \epsilon\left(1-\frac{\left|E_{g, 0}\right|}{\left|E_{g}\right|}\right)$

where $E_{k}$ and $E_{g}$ are the kinetic and gravitational energies, respectively, the 0 index indicates the initial value, i.e. at the start of the collapse/fragmentation, and $\epsilon$ is the fraction of the gravitational energy which is converted into kinetic energy. Note that as $\left|E_{g}\right|$ becomes increasingly larger during the collapse, $\epsilon=1$ translates into $\alpha_{\mathrm{vir}} \rightarrow 2$, which is expected if all the gravitational energy were to be converted into kinetic energy. A mean $a_{\text {eff, } 0} \sim 0.26 \pm 0.02 \mathrm{~km} \mathrm{~s}^{-1}$ is evaluated from Fig. 17 (as discussed in Sect. 5.1). By estimating each energy term of Eq. (8) for every core showing an increase of velocity dispersion one can determine which value of $\epsilon$ is required to reproduce the observed $\alpha_{\text {vir }}$ value. Assuming that the cores of initial diameter $\lambda_{\text {core }}$ result from the fragmentation of a uniform density transonic filament of initial velocity dispersion $a_{\mathrm{eff}, 0}$, one can rewrite Eq. (8) as:

$\alpha_{\mathrm{vir}}=\frac{3}{\beta G} \frac{R a_{\mathrm{eff}, 0}^{2}}{M}+2 \epsilon\left(1-\frac{2 \beta_{0}}{\beta} \frac{R}{\lambda_{\text {core }}}\right)$

where $\beta=\frac{3-k_{\rho}}{5-2 k_{\rho}}$ is a factor depending on the power law index $k_{\rho}$ of the density profile of the collapsing core, and $\beta_{0}$ is its initial value before the onset of collapse (see Appendix F for a more detailed derivation). For simplicity, we assumed an initially flat density profile (i.e. $\beta_{0}=3 / 5$ ), and $k_{\rho}=2$ once collapse begins (i.e. $\beta=1$ ). Figure 19 plots the energy conversion efficiency $\epsilon$ against the virial ratio for every core for both the combined JVLA/GBT data and JVLA-only data. On this plot, only starless cores are included, as proto-stellar cores, or cores at filament junctions are subject to extra energy sources that will affect the estimate of $\epsilon$. One can see that a conversion efficiency between 20 and $50 \%$ can explain most of the observed velocity dispersion increases towards starless cores. The JVLA-only data 


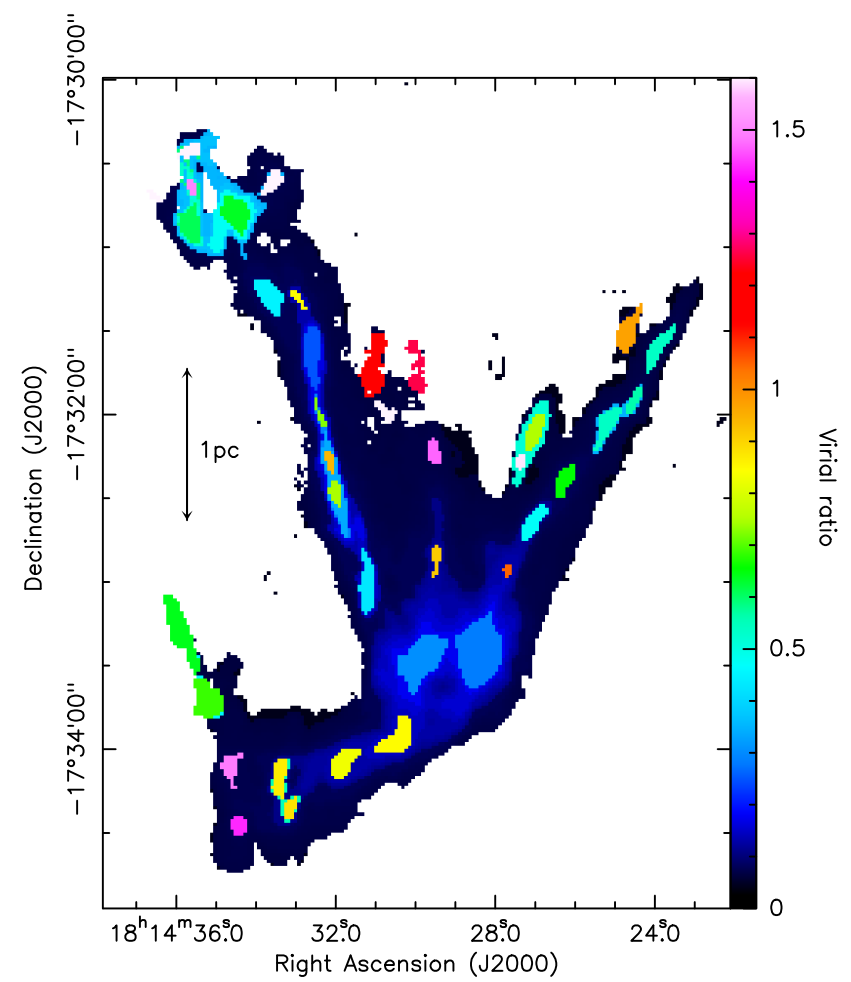

Fig. 18. Virial ratio map of all identified dendrogram structures.

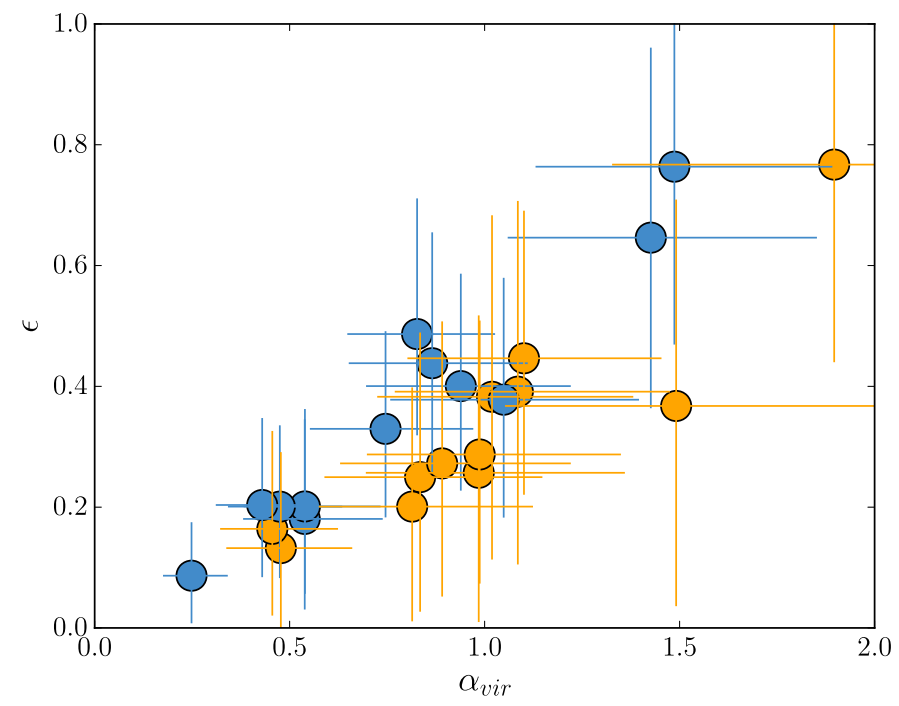

Fig. 19. Observed virial ratio plotted against the energy conversion efficiency of gravitational energy into kinetic energy for the combined JVLA/GBT data (in blue), and the JVLA-only data (in orange). Only starless along the filaments are plotted, as proto-stellar or hub centre cores are subject to extra energy source that will effect the estimate of $\epsilon$. Errors were propagated using a Monte Carlo error propagation method.

points (orange circles), believed to be less contaminated by foreground/background emission to the cores, are clustered around a median efficiency of $35 \%$.

Klessen \& Hennebelle (2010) proposed a theory according to which accretion is capable of driving turbulent motions in galaxies on all scales, finding an $\epsilon$ value of a few percent when considering both galaxy and molecular cloud sized structures. Clarke et al. (2017) evaluate the efficiency required to reproduce a given velocity dispersion due to accretion driven turbulence in filament structures. They find an $\epsilon=5-10 \%$ is sufficient to drive a $\sigma_{1 \mathrm{D}} \sim 0.23 \mathrm{~km} \mathrm{~s}^{-1}$, a consistent efficiency to that found by Heitsch (2013). While these are theoretical estimates, our observationally derived value of $\sim 35 \%$ is somewhat larger. This could point towards an even more important role of gravity-driven turbulence than previously thought (see also Traficante et al. 2018; Ballesteros-Paredes et al. 2017).

\subsection{Origin of the radial velocity gradients}

Two of the four SDC13 filaments exhibit strong radial velocity gradients, the largest of which in the combined data set reaches $1.5 \mathrm{~km} \mathrm{~s}^{-1} \mathrm{pc}^{-1}$, an order of magnitude larger than in the remaining two filaments. In the JVLA-only data, although the morphology of the centroid line-of-sight velocity is the same as in the combined data, the magnitude of the radial velocity gradients double, approaching $\sim 3.0 \mathrm{~km} \mathrm{~s}^{-1} \mathrm{pc}^{-1}$ in both the north-east and south-east filaments. The physical origin of such gradients is unclear as they could be the result of accretion/compression, rotation, shear, or a combination of these. It is important to realise that, given the measured velocity gradients (up to $3.0 \mathrm{~km} \mathrm{~s}^{-1} \mathrm{pc}^{-1}$ ), the crossing time of the filaments is $\sim 0.3-0.4 \mathrm{Myr}$, which is a factor of $\sim 3$ lower than the estimated age of the filaments. This implies that, whatever the origin of the gradient is, it cannot be of disruptive nature or the filaments would already have dispersed. This therefore excludes shear motions as a possible origin. Here, we are going to investigate if the magnitude of the gradients are compatible with gravity and/or rotation.

For gravity to be responsible for radial velocity gradients in filaments, large-scale accretion into a plane is the only option as axisymmetric accretion would not produce any velocity gradients. Assuming that the filaments are infinitely long, then one can estimate the acceleration of a piece of gas at a radius $r$ (Palmeirim et al. 2013), as:

$\mathrm{v}_{\mathrm{r}}=2 \sqrt{G M_{\text {line }} \ln \left(\frac{r_{\text {initial }}}{r}\right)}$.

Substituting for the mean observed $M_{\text {line }}$ of SDC13 we may calculate the $r_{\text {initial }}$ that would produce the observed velocity $\left(\sim 0.3 \mathrm{~km} \mathrm{~s}^{-1}\right)$ at a distance of $0.20 \mathrm{pc}$ from the spine of the filament. By doing so we obtain $r_{\text {initial }} \simeq 0.21 \mathrm{pc}$. The associated dynamical timescale is $3.3 \times 10^{4} \mathrm{yr}$, nearly two orders of magnitude shorter than our age estimates for the filaments. This suggests that gravity alone is not the origin of the observed radial velocity gradients in SDC13. As proposed by Arzoumanian et al. (2013), turbulence generated by the accretion of matter onto filaments can provide an additional kinetic pressure that can eventually counteract the pull of gravity, and slow down the filament radial contraction, even though such a process might not be efficient enough (Seifried \& Walch 2015). Magnetic fields can also contribute to slow down radial contraction, either when they run parallel to the main axis of filaments (Seifried \& Walch 2015), or when in a helical configuration (Fiege \& Pudritz 2000). However, $B$ fields are often observed to be perpendicular to the main axis of the star-forming filaments (e.g. Palmeirim et al. 2013; Cox et al. 2016), therefore leaving the radial contraction unaffected.

We now investigate whether rotation could explain these observed gradients. Recchi et al. (2014) simulate the rotation about the main axis of filaments in equilibrium. They predict the radial velocity gradient required for rotation to stabilise filaments with varying $M_{\text {line }}$ and temperature profiles against gravitational instabilities. Following their calculations for a mean 
SDC13 filament mass of $319 M_{\odot}$, mean filament length and width of 2.11 and $0.26 \mathrm{pc}$, respectively (hence central density of $4.8 \times 10^{-17} \mathrm{~kg} \mathrm{~m}^{-3}$ ), and uniform temperature profile (given we assume a constant temperature everywhere), a radial velocity gradient of $1.42 \mathrm{~km} \mathrm{~s}^{-1} \mathrm{pc}^{-1}$ is required for rotation to halt the fragmentation of SDC13. Despite this being larger than the mean radial velocity gradient across the south-east filament (and less than that across the north-east filament) it is clear from the core extraction that there is indeed fragmentation along both filaments. Hence, we conclude that the observed radial velocity gradient cannot be solely due to rotation as the fragmentation was not halted. The model also only considers equilibrium filaments, which seems to be an unrealistic assumption to make for such a dynamic system as SDC13.

Therefore, we believe that the most likely origin for the observed radial velocity gradients is compression. We speculate that an external site of active star formation, clearly seen in Fig. 1 within $1 \mathrm{pc}$ proximity to the north-east filament on the sky (centred on 18:14:33.5-17:33:30.0) may be compressing the north-east and south-east filaments of SDC13, influencing the radial velocity field and their density structures. The WISE catalogue of HII region sources (Anderson et al. 2014) reveals a HII region they term as being radio-quiet (called G013.170-00.097) coinciding with this external star formation site, however there is no kinematic information available. Further to this, large scale ${ }^{13} \mathrm{CO}(1-0)$ and $\mathrm{C}^{18} \mathrm{O}(1-0)$ emission from the IRAM $30 \mathrm{~m}$ telescope seem to show a cavity in emission corresponding to this region (Williams et al., in prep.), consistent with the clearing of material due to feedback effects. We find no maser emission such as water (Walsh et al. 2008; Purcell et al. 2012) nor methanol (Breen et al. 2012) around the position of the HII region. Other works have shown the influence of external feedback on the density structure of star-forming filaments (Peretto et al. 2012; Tremblin et al. 2014).

\subsection{Origin of the longitudinal velocity gradients}

As mentioned in Sect. 4.2.1, the SDC13 filaments exhibit a complex oscillating longitudinal velocity profile which is at least partially correlated to their column density structure. In their simulations of turbulent cloud evolution, Smith et al. (2016) find very similar looking filament velocity profiles (see their Fig. 6). However, they argue that they do not see any clear correlation between the velocity fluctuations and the location of density peaks, concluding that the velocity fluctuations are the result of transient motions generated by the turbulent field in which the filaments are immerged. While we might be partly witnessing such effect in some of the SDC13 filaments, we do observe that $\sim 63 \%$ of the SDC13 cores are located at a peak of velocity gradient as expected for cores accreting from their parent filaments.

One specificity of SDC13 is its hub morphology, suggestive of converging gravity-driven flows towards the filament junction. In order to evaluate if the hub morphology has an impact on the density and velocity profiles of SDC13, for each position we computed the amplitude of the acceleration following:

$\left|\boldsymbol{a}_{j}\right|=\left|-\sum_{i \neq j} \frac{G m_{i}}{r_{i, j}^{3}} \boldsymbol{r}_{i, j}\right|$

where indices $i$ and $j$ correspond to given pixels in our SDC13 column density map, $m_{i}$ is the gas mass at pixel $i$, and $r_{i, j}$ is the distance between pixels $i$ and $j$. Observationally, one only has

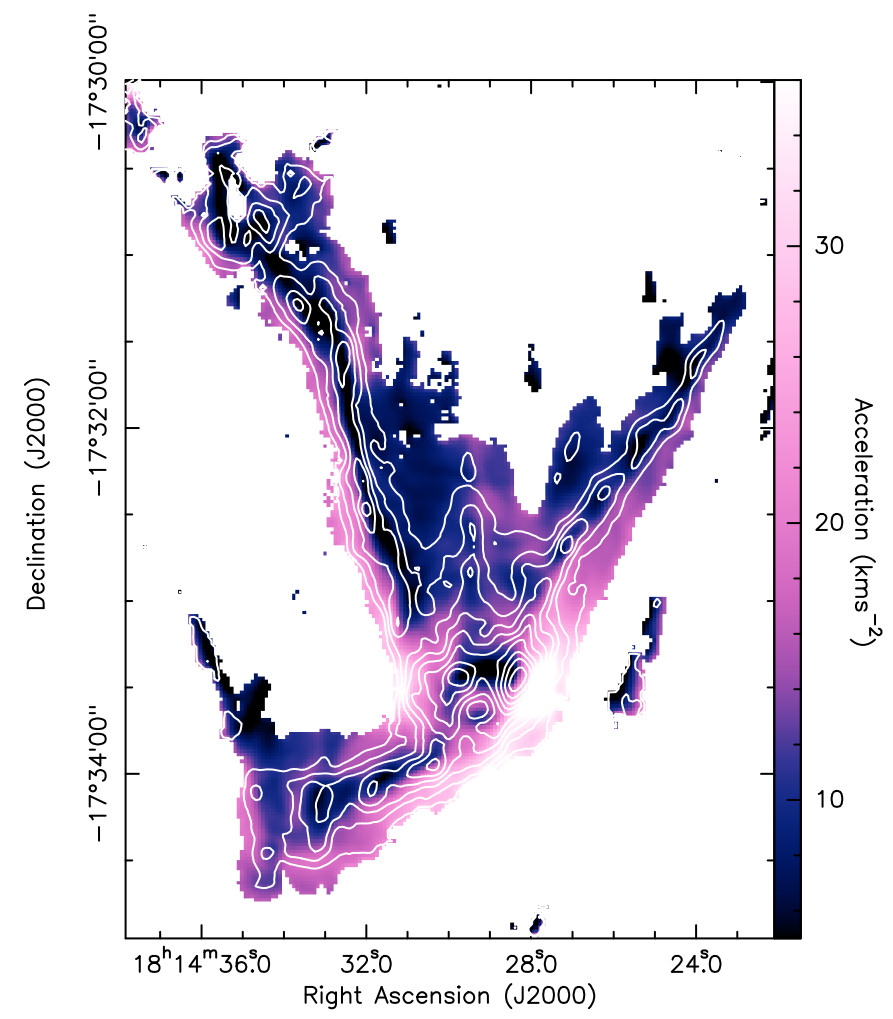

Fig. 20. Map of gravitational acceleration in SDC13 in units of $1 \times 10^{-12} \mathrm{~km} \mathrm{~s}^{-2}$. Mass was calculated from the $\mathrm{H}_{2}$ column density map in the middle panel of Fig. 5. Contours are of the $\mathrm{H}_{2}$ column density in Fig. 5, plotted in $1 \times 10^{22} \mathrm{~cm}^{-2}$ steps, from $2 \times 10^{22} \mathrm{~cm}^{-2}$ to $12 \times 10^{22} \mathrm{~cm}^{-2}$.

access to the $2 \mathrm{D}$ projected quantities, and acceleration estimated that way can only provide trend indications and order of magnitude estimates. Figure 20 shows the acceleration map obtained from the SDC13 $\mathrm{H}_{2}$ column density map. As expected, the accelerations along the filament spine are at a minimum due to the axisymmetric nature of gravity in such systems. The acceleration appears to peak around the filament hub junctions and the edges of some filaments, reminiscent of the behaviour seen in the velocity dispersion map in Fig. 4. More interestingly, we have constructed acceleration profiles along each filament. These are shown in the first row of Fig. 21. We observe oscillations of the acceleration and an increase of the acceleration towards the hub centre which are reminiscent of the velocity structure. In order to check if this behaviour is due to the presence of cores or to the hub morphology, we constructed another acceleration map with uniform column density, effectively removing the presence of density fluctuations. The second row of Fig. 21 shows such profiles. One can see that, for the two main filaments (northeast and north-west), the acceleration profiles remain mostly unchanged, demonstrating that the hub morphology is the main driver of the acceleration fluctuation we observed, and therefore the driver of the SDC13 kinematics as a whole. Another interesting point is given by the last row of Fig. 21 where the gradients of the acceleration is provided. We notice here that the strongest acceleration gradient peak is associated with the most massive core within SDC13. Gradients of acceleration represent accumulation points and are therefore privileged locations for the formation of massive cores. The systematic presence of massive cores near the hub centre could therefore be a direct consequence of large acceleration gradients generated by the hub morphology. 


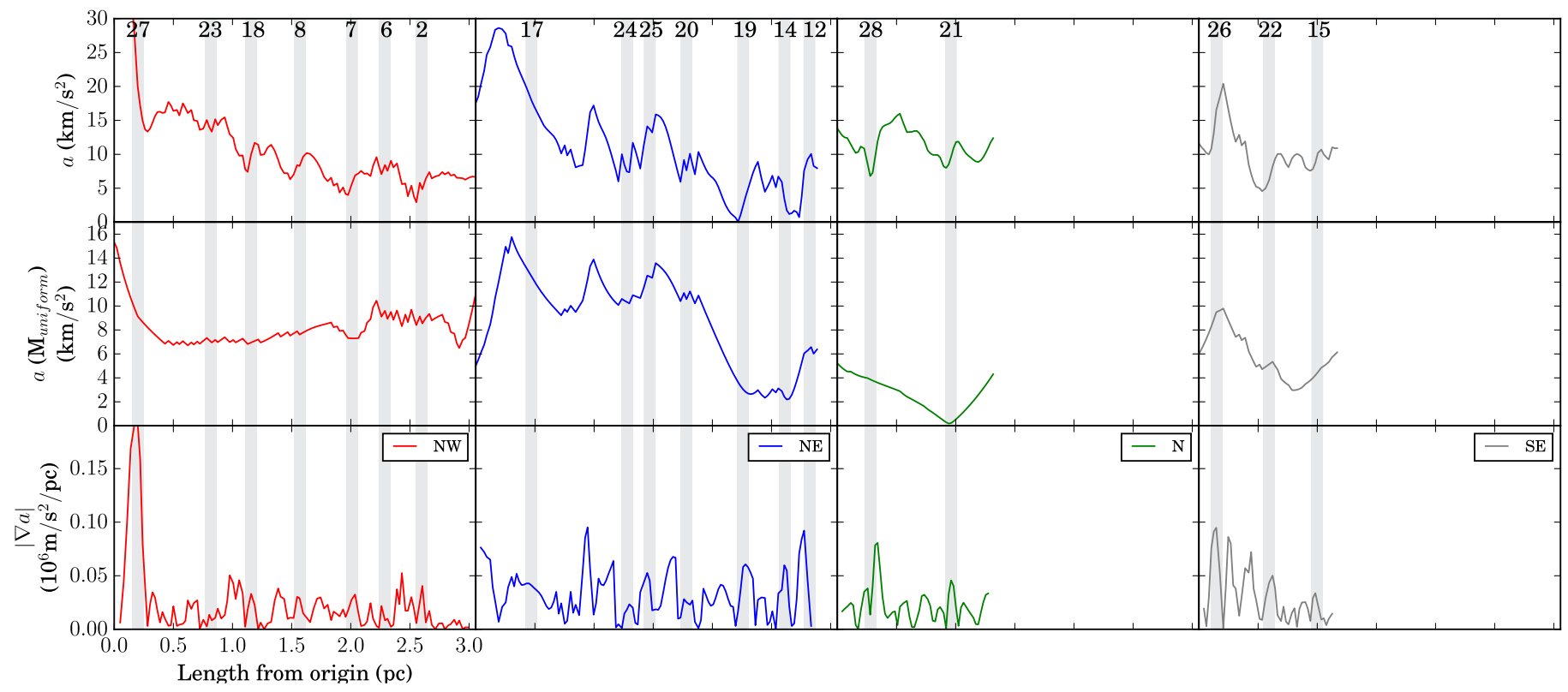

Fig. 21. Profiles along the spines, where each column denotes a different filamentary arm, as labelled (north-west in red, north-east in blue, north in green and south-east in grey). The origin of each of the spines was defined to begin at the heart of the hub. First row: acceleration due to gravity in SDC13 where the mass was calculated using the $\mathrm{H}_{2}$ column density map, while a uniform, mean cloud mass was used for the acceleration in the second row. Third row: absolute gradient of the acceleration from row three evaluated at each position over the mean core size of $\sim 0.1 \mathrm{pc}$. The vertical shaded regions correspond to the positions of the cores along each of the filaments, are $\sim 0.1 \mathrm{pc}$ wide and overplotted by the core ID number in Table 2.

\section{Summary and conclusions}

We have conducted a study of the SDC13 infrared dark hub filament system using the rotation inversion transition of $\mathrm{NH}_{3}(1,1)$ and $\mathrm{NH}_{3}(2,2)$. The fitting of the hyperfine structure of these lines allowed the construction of centroid velocity, velocity dispersion and $\mathrm{H}_{2}$ column density maps. These maps were used to identify both core structures and filament spines, allowing us to characterise their kinematics. We find that all filaments are supercritical, with an average transonic non-thermal velocity dispersion, and that core spacing is typically regular along them $(\sim 0.37 \pm 0.16 \mathrm{pc})$. Using semi-analytical models of nonequilibrium filaments (Clarke et al. 2016, 2017) we determine that the filaments are a few Myr old, consistent with the SDC13 dynamical timescale derived by Peretto et al. (2014). We find that the large radial velocity gradients across two of the four filament cannot be due to gravity nor rotation, but most likely due to the compression caused by a nearby HII region. We also find that the velocity dispersion increases towards $73 \%$ of the identified cores, which we interpret as the result of the accumulation of matter due to the filament fragmentation process itself. We derive an average gravitational to kinetic energy conversion efficiency within these cores of $\epsilon \simeq 35 \%$, larger than theoretical values published in the literature. Finally, we propose that the presence of massive cores near the filament junction is due to the large acceleration gradients produced by the hub morphology.

All elements put together, we propose a scenario for the evolution of the SDC13 hub-filament system in which filaments first form as post-shock structures in a supersonic turbulent flow. As a result of the turbulent energy dissipation in the shock, the dense gas within the filaments is initially mostly sub-sonic. Then gravity takes over and starts shaping the evolution of the hub, both fragmenting filaments and pulling the gas towards the centre of the gravitational well. By doing so, gravitational energy is converted into kinetic energy in both local (cores) and global (hub centre) potential well minima, generating more massive cores at the hub centre as a result of larger acceleration gradients.

Acknowledgements. GMW gratefully acknowledges the support of an STFC postgraduate studentship. NP wishes to acknowledge support from STFC under grant number ST/N000706/1 and ST/M000893/1. We thank the anonymous referee whose report helped improve the quality of this paper. This research made use of APLpy, an open-source plotting package for Python hosted at http://aplpy.github. com.

\section{References}

Anderson, L. D., Bania, T. M., Balser, D. S., et al. 2014, ApJS, 212, 1 André, P., Men'shchikov, A., Bontemps, S., et al. 2010, A\&A, 518, L102 Arzoumanian, D., André, P., Didelon, P., et al. 2011, A\&A, 529, L6 Arzoumanian, D., André, P., Peretto, N., \& Könyves, V. 2013, A\&A, 553, A119 Ballesteros-Paredes, J., Vázquez-Semadeni, E., Palau, A., \& Klessen, R. S. 2017, MNRAS, submitted [arXiv: 1710.07384]

Barranco, J. A., \& Goodman, A. A. 1998, ApJ, 504, 207

Barrett, A. H., Ho, P. T. P., \& Myers, P. C. 1977, ApJ, 211, L39

Bergin, E. A., \& Langer, W. D. 1997, ApJ, 486, 316

Bertoldi, F., \& McKee, C. F. 1992, ApJ, 395, 140

Beuther, H., \& Steinacker, J. 2007, ApJ, 656, L85

Beuther, H., Ragan, S. E., Johnston, K., et al. 2015, A\&A, 584, A67

Breen, S. L., Ellingsen, S. P., Caswell, J. L., et al. 2012, MNRAS, 426, 2189

Butler, M. J., \& Tan, J. C. 2009, ApJ, 696, 484

Carey, S. J., Noriega-Crespo, A., Mizuno, D. R., et al. 2009, PASP, 121, 76

Caselli, P., Benson, P. J., Myers, P. C., \& Tafalla, M. 2002, ApJ, 572, 238

Cheung, A. C., Rank, D. M., Townes, C. H., Thornton, D. D., \& Welch, W. J. 1968, Phys. Rev. Lett., 21, 1701

Churchwell, E., Babler, B. L., Meade, M. R., et al. 2009, PASP, 121, 213

Clarke, S. D., \& Whitworth, A. P. 2015, MNRAS, 449, 1819

Clarke, S. D., Whitworth, A. P., \& Hubber, D. A. 2016, MNRAS, 458, 319

Clarke, S. D., Whitworth, A. P., Duarte-Cabral, A., \& Hubber, D. A. 2017, MNRAS, 468, 2489

Cox, N. L. J., Arzoumanian, D., André, P., et al. 2016, A\&A, 590, A110

Dirienzo, W. J., Brogan, C., Indebetouw, R., et al. 2015, ApJ, 150, 159

Duarte-Cabral, A., Chrysostomou, A., Peretto, N., et al. 2012, A\&A, 543, A140

Egan, M. P., Shipman, R. F., Price, S. D., et al. 1998, ApJ, 494, L199 
Federrath, C., Rathborne, J. M., Longmore, S. N., et al. 2016, ApJ, 832, 143

Fernández-López, M., Arce, H. G., Looney, L., et al. 2014, ApJ, 790, L19

Fiege, J. D., \& Pudritz, R. E. 2000, MNRAS, 311, 85

Fuller, G. A. \& Myers, P. C. 1992, ApJ, 384, 523

Girichidis, P., Federrath, C., Banerjee, R., \& Klessen, R. S. 2011, MNRAS, 413, 2741

Goldsmith, P. F., \& Langer, W. D. 1999, ApJ, 517, 209

Goodman, A. A., Barranco, J. A., Wilner, D. J., \& Heyer, M. H. 1998, ApJ, 504, 223

Gunther-Mohr, G. R. 1954, Ph.D. thesis, Columbia University

Hacar, A., \& Tafalla, M. 2011, A\&A, 533, A34

Hacar, A., Kainulainen, J., Tafalla, M., Beuther, H., \& Alves, J. 2016, A\&A, 587, A97

Harju, J., Walmsley, C. M., \& Wouterloot, J. G. A. 1993, A\&AS, 98, 51

Heitsch, F. 2013, ApJ, 769, 115

Henshaw, J. D., Caselli, P., Fontani, F., et al. 2016, MNRAS, 463, 146

Ho, P. T. P., \& Townes, C. H. 1983, ARA\&A, 21, 239

Inutsuka, S.-I., \& Miyama, S. M. 1992, ApJ, 388, 392

Kirk, H., Myers, P. C., Bourke, T. L., et al. 2013, ApJ, 766, 115

Klessen, R. S., \& Hennebelle, P. 2010, A\&A, 520, A17

Klessen, R. S., Ballesteros-Paredes, J., Vázquez-Semadeni, E., \& Durán-Rojas, C. 2005, ApJ, 620, 786

Könyves, V., André, P., Men'shchikov, A., et al. 2015, A\&A, 584, A91

Kukolich, S. G. 1967, Phys. Rev., 156, 83

Li, D., Goldsmith, P. F., \& Menten, K. 2003, ApJ, 587, 262

Liu, H. B., Ho, P. T. P., Wright, M. C. H., et al. 2013, ApJ, 770, 44

Lu, X., Zhang, Q., Liu, H. B., Wang, J., \& Gu, Q. 2014, ApJ, 790, 84

Maret, S., Faure, A., Scifoni, E., \& Wiesenfeld, L. 2009, MNRAS, 399, 425

Marsh, K. A., Kirk, J. M., André, P., et al. 2016, MNRAS, 459, 342

Masters, J., Garwood, B., Langston, G., \& Shelton, A. 2011, in Astronomical Data Analysis Software and Systems XX, eds. I. N. Evans, A. Accomazzi, D. J. Mink, \& A. H. Rots, ASP Conf. Ser., 442, 127

McGuire, C., Fuller, G. A., Peretto, N., et al. 2016, A\&A, 594, A118

McMullin, J. P., Waters, B., Schiebel, D., Young, W., \& Golap, K. 1992, ASP Conf. Ser., 376, 127

Miyama, S. M., Nakamoto, T., Kikuchi, N., et al. 1994, The Stability of Circumstellar Disks (Cambridge: Cambridge University Press)

Molinari, S., Swinyard, B., Bally, J., et al. 2010, PASP, 122, 314

Morgan, L. K., Moore, T. J. T., Allsopp, J., \& Eden, D. J. 2013, MNRAS, 428, 1160

Myers, P. C. 1983, ApJ, 270, 105

Myers, P. C. 2009, ApJ, 700, 1609

Ostriker, J. 1964, ApJ, 140, 1056

Padoan, P., Nordlund, Å., Rögnvaldsson, Ö. E., \& Goodman, A. 2001, in From Darkness to Light: Origin and Evolution of Young Stellar Clusters, eds. T. Montmerle, \& P. André, ASP Conf. Ser., 243, 279
Palmeirim, P., André, P., Kirk, J., et al. 2013, A\&A, 550, A38

Panopoulou, G. V., Tassis, K., Goldsmith, P. F., \& Heyer, M. H. 2014, MNRAS, 444, 2507

Panopoulou, G. V., Psaradaki, I., Skalidis, R., Tassis, K., \& Andrews, J. J. 2017, MNRAS, 466, 2529

Perault, M., Omont, A., Simon, G., et al. 1996, A\&A, 315, L165

Peretto, N., \& Fuller, G. A. 2009, A\&A, 505, 405

Peretto, N., Fuller, G. A., Plume, R., et al. 2010, A\&A, 518, L98

Peretto, N., André, P., Könyves, V., et al. 2012, A\&A, 541, A63

Peretto, N., Fuller, G. A., Duarte-Cabral, A., et al. 2013, A\&A, 555, A112

Peretto, N., Fuller, G. A., André, P., et al. 2014, A\&A, 561, A83

Pillai, T., Wyrowski, F., Carey, S. J., \& Menten, K. M. 2006, A\&A, 450, 569

Pillai, T., Kauffmann, J., Wyrowski, F., et al. 2011, A\&A, 530, A118

Pineda, J. E., Goodman, A. A., Arce, H. G., et al. 2010, ApJ, 712, L116

Pineda, J. E., Offner, S. S. R., Parker, R. J., et al. 2015, Nature, 518, 213

Polychroni, D., Schisano, E., Elia, D., et al. 2013, ApJ, 777, L33

Pon, A., Toalá, J. A., Johnstone, D., et al. 2012, ApJ, 756, 145

Purcell, C. R., Longmore, S. N., Walsh, A. J., et al. 2012, MNRAS, 426, 1972

Ragan, S. E., Bergin, E. A., \& Wilner, D. 2011, ApJ, 736, 163

Rathborne, J. M., Jackson, J. M., \& Simon, R. 2006, ApJ, 641, 389

Recchi, S., Hacar, A., \& Palestini, A. 2014, MNRAS, 444, 1775

Rosolowsky, E. W., Pineda, J. E., Kauffmann, J., \& Goodman, A. A. 2008, ApJ, 679, 1338

Salji, C. J., Richer, J. S., Buckle, J. V., et al. 2015, MNRAS, 449, 1782

Schisano, E., Rygl, K. L. J., Molinari, S., et al. 2014, ApJ, 791, 27

Schneider, S. \& Elmegreen, B. G. 1979, ApJS, 41, 87

Schneider, N., Csengeri, T., Hennemann, M., et al. 2012, A\&A, 540, L11

Seifried, D., \& Walch, S. 2015, MNRAS, 452, 2410

Shirley, Y. L. 2015, PASP, 127, 299

Simon, R., Rathborne, J. M., Shah, R. Y., Jackson, J. M., \& Chambers, E. T. 2006, ApJ, 653, 1325

Smith, R. J., Glover, S. C. O., Klessen, R. S., \& Fuller, G. A. 2016, MNRAS, 455,3640

Teixeira, P. S., Takahashi, S., Zapata, L. A., \& Ho, P. T. P. 2016, A\&A, 587, A47

Toalá, J. A., Vázquez-Semadeni, E., \& Gómez, G. C. 2012, ApJ, 744, 190

Traficante, A., Fuller, G. A., Smith, R. J., et al. 2018, MNRAS, 473, 4975

Tremblin, P., Schneider, N., Minier, V., et al. 2014, A\&A, 564, A106

Ungerechts, H., Winnewisser, G., \& Walmsley, C. M. 1986, A\&A, 157, 207

Vázquez-Semadeni, E., Gómez, G. C., Jappsen, A.-K., Ballesteros-Paredes, J., \& Klessen, R. S. 2009, ApJ, 707, 1023

Vázquez-Semadeni, E., González-Samaniego, A., \& Colín, P. 2017, MNRAS, 467, 1313

Walsh, A. J., Lo, N., Burton, M. G., et al. 2008, PASA, 25, 105

Whitworth, A. P., \& Ward-Thompson, D. 2001, ApJ, 547, 317

Zhang, Q., Wang, Y., Pillai, T., \& Rathborne, J. 2009, ApJ, 696, 268 


\section{Appendix A: Channel map}

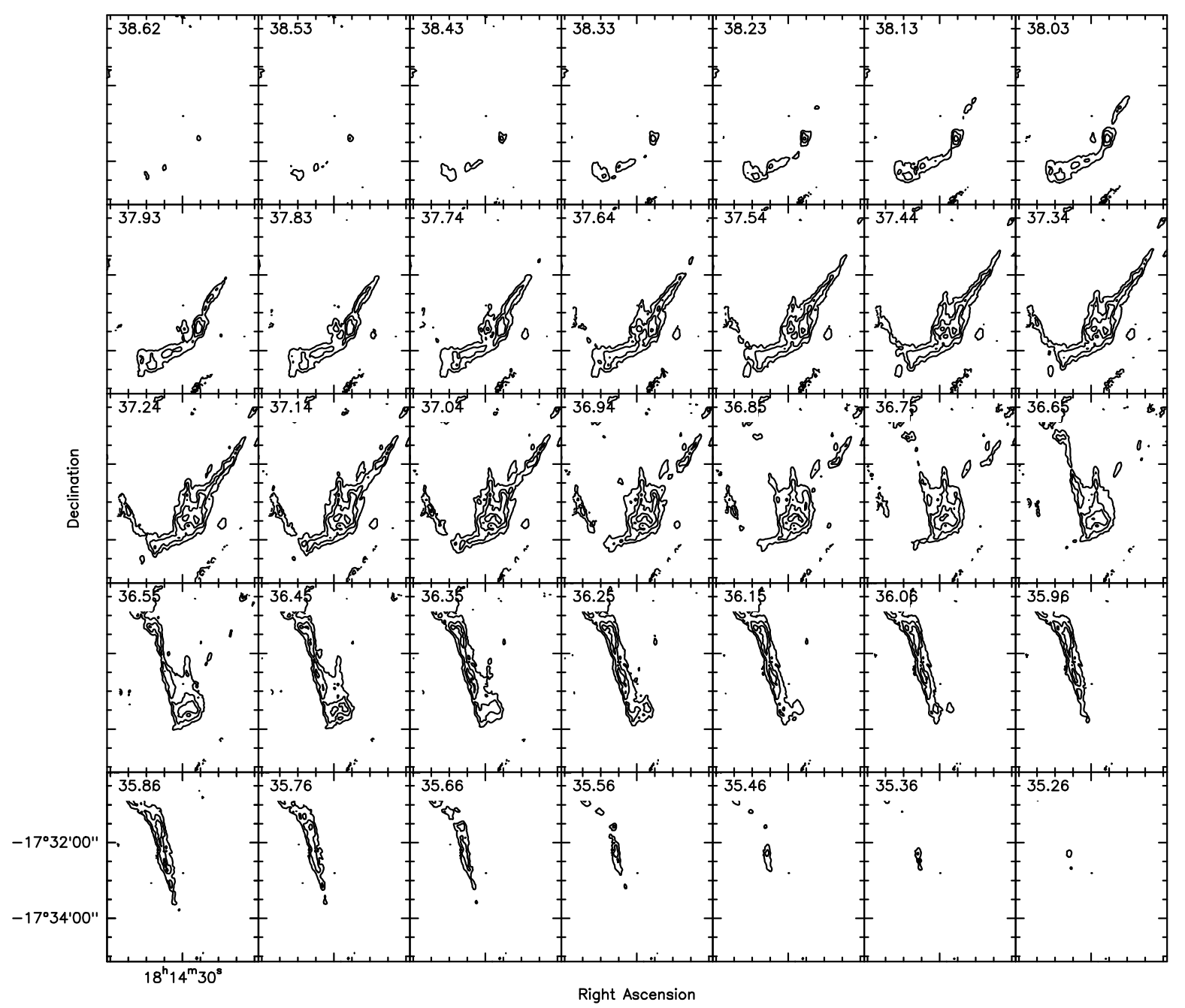

Fig. A.1. Channel map of the main transition of $\mathrm{NH}_{3}(1,1)$ in the model combined data cube. Contour levels are placed at $0.05 \mathrm{Jy}$ beam ${ }^{-1}$ steps, from $0.05 \mathrm{Jy}_{\text {beam }}^{-1}$ to $0.2 \mathrm{Jy} \mathrm{beam}^{-1}$. The channel velocity in $\mathrm{km} \mathrm{s}^{-1}$ is plotted in the top left corner of each panel. Right Ascension and Declination are in the $\mathrm{J} 2000$ epoch.

Here we show the channel map of the main $\mathrm{NH}_{3}(1,1)$ emission in SDC13.

\section{Appendix B: Opacity, temperature and column density}

Here we present a more in depth discussion on the calculation of the opacity, temperature and column density directly from the relative intensities of the $\mathrm{NH}_{3}(1,1)$ and $\mathrm{NH}_{3}(2,2)$ lines.

\section{B.1. Opacity}

The optical depth defines the extent to which the $\mathrm{NH}_{3}$ gas is opaque or transparent, and was solved numerically (Barrett et al. 1977),

$\frac{\Delta T_{a}(1,1, m)}{\Delta T_{a}(1,1, s)}=\frac{1-\mathrm{e}^{-\tau(1,1, m)}}{1-\mathrm{e}^{-\mathrm{a} \tau(1,1, m)}}$,

where the parameters $\Delta T_{a}(1,1, m)$ and $\Delta T_{a}(1,1, s)$ refer to the observed peak spectra of the $\mathrm{NH}_{3}(1,1)$ main (denoted by the $m$ ) and satellite (denoted by the $s$ ) components, which we take from the model hyperfine structure fit. The expected relative intensities between the main and inner satellite lines is denoted by $a$. The opacity of the main $\mathrm{NH}_{3}(1,1)$ line is denoted by $\tau(1,1, m)$. This opacity is converted to the total opacity, $\tau(1,1, t)$, by dividing by the relative strength of 0.502 ( $\mathrm{Li}$ et al. 2003).

The opacity of $\mathrm{NH}_{3}(2,2)$ is calculated similarly (Barrett et al. 1977),

$\frac{\Delta T_{a}(1,1, m)}{\Delta T_{a}(2,2, m)}=\frac{1-\mathrm{e}^{-\tau(1,1, m)}}{1-\mathrm{e}^{-\tau(2,2, m)}}$,

where $\Delta T_{a}(2,2, m)$ and $\tau(2,2, m)$ are the observed peak of the spectra and the opacity of the $\mathrm{NH}_{3}(2,2)$ main component, respectively. This opacity is converted to the total opacity, $\tau(2,2, t)$ of $\mathrm{NH}_{3}(2,2)$ by dividing by the relative strength of 0.796 ( $\mathrm{Li}$ et al. 2003).

\section{B.2. Temperature}

The rotational temperature, which describes the relative populations of the $\mathrm{NH}_{3}(1,1)$ and $\mathrm{NH}_{3}(2,2)$ levels, was found using the following equation (Ungerechts et al. 1986), 

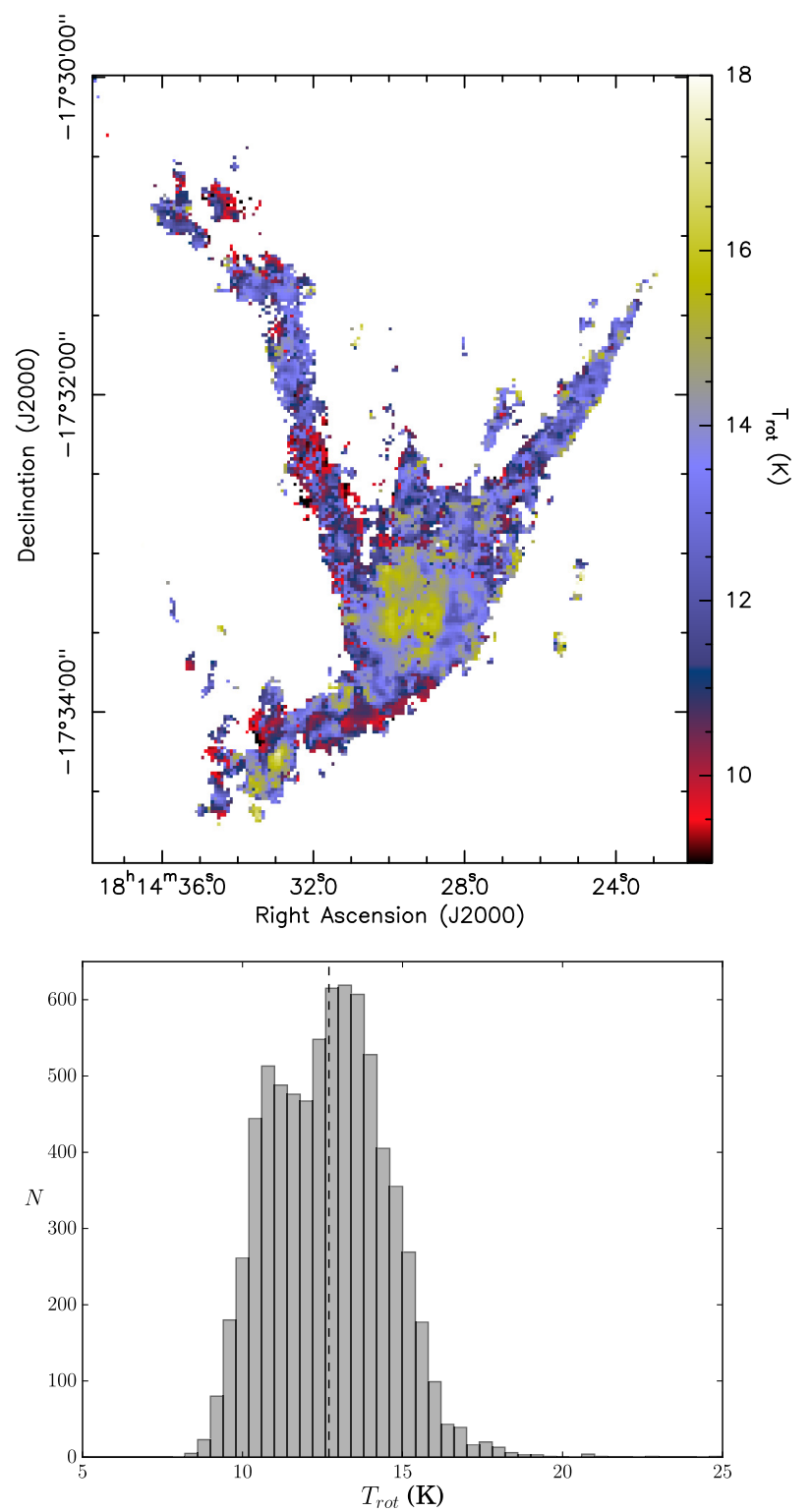

Fig. B.1. Top: map of the rotational temperature calculated using Eq. (B.3). It is clear that the coverage of the image is limited to that of the weaker $\mathrm{NH}_{3}(2,2)$ line. No correlation is found between temperature and core position. Bottom: histogram of the $T_{\text {rot }}$ map in the top panel. Vertical dashed line indicates the median value of $12.7 \mathrm{~K}$. The standard deviation is $1.8 \mathrm{~K}$.

$T_{\text {rot }}=-T_{0} / \ln \left(\frac{9}{20} \frac{\tau(2,2, t)}{\tau(1,1, t)}\left[\frac{\Delta v_{2,2}}{\Delta v_{1,1}}\right]\right)$,

where $\tau(2,2, t)$ and $\tau(1,1, t)$ are the total optical depths, and $\Delta v_{2,2}$ and $\Delta v_{1,1}$ are the velocity widths of the $\mathrm{NH}_{3}(2,2)$ and $\mathrm{NH}_{3}(1,1)$ lines, respectively, and $T_{0}=\left(E_{2,2}-E_{1,1}\right) / k=41 \mathrm{~K}$, where $E_{1,1}$ and $E_{2,2}$ are the energies of the $\mathrm{NH}_{3}(1,1)$ and $\mathrm{NH}_{3}(2,2)$ lines, respectively. The resulting $T_{\text {rot }}$ map is shown in the top panel of Fig. B.1, with its corresponding histogram in the bottom panel.
We find no correlation between temperature and core positions, and only notice a very mild warming of material at the filament hub junctions.

As outlined in Sect. 3.1.1, the $\mathrm{NH}_{3}(2,2)$ emission is approximately 6 times weaker than that of $\mathrm{NH}_{3}(1,1)$, resulting in $42 \%$ less coverage in the $\mathrm{NH}_{3}(2,2)$ maps, and hence, the temperature maps. This limits the calculation of the column density of the cloud to this lesser coverage. To overcome this hindrance, we took a median $T_{\text {rot }}$ across the entire cloud of $12.7 \mathrm{~K}$ (e.g. Morgan et al. 2013). We discounted using the full temperature map whilst setting the median in the $\mathrm{NH}_{3}(1,1)$ regions of emission only, as this caused edge effects in the resulting column density.

\section{B.3. Column density}

With opacity and temperature, we calculated the total column density of $\mathrm{NH}_{3}$ and $\mathrm{H}_{2}$. Firstly, the column density of the $\mathrm{NH}_{3}(1,1)$ upper level only was calculated (Goldsmith \& Langer 1999),

$N\left[\mathrm{NH}_{3}(1,1)\right]=\frac{8 k \pi v_{1,1}^{2}}{A_{\mathrm{u}, 1} h c^{3}} \int_{-\infty}^{\infty} T_{b} \mathrm{~d} v\left(\frac{\tau(1,1, t)}{1-\mathrm{e}^{-\tau(1,1, t)}}\right)$,

where $k$ is the Boltzmann's constant, $v_{1,1}$ is the rest frequency of the $\mathrm{NH}_{3}(1,1)$ line, $A_{u l}$ is the Einstein Coefficient for spontaneous emission, $h$ is the Planck constant, $c$ is the speed of light, $\tau(1,1, t)$ is the total opacity of the $\mathrm{NH}_{3}(1,1)$ line, and $\int_{-\infty}^{\infty} T_{b} \mathrm{~d} v$ is the integrated intensity of $\mathrm{NH}_{3}(1,1)$ in Fig. 4a. Beam information has been cancelled out under the assumption that the source fills the beam.

Assuming local thermodynamic equilibrium (LTE), where the rotational temperature is assumed to equal the excitation temperature, we converted $N\left[\mathrm{NH}_{3}(1,1)\right]$ into the total column density of Ammonia, $N\left(\mathrm{NH}_{3}\right)$, using (Goldsmith \& Langer 1999),

$N\left(\mathrm{NH}_{3}\right)=\frac{N\left[\mathrm{NH}_{3}(1,1)\right]}{g_{1,1}} \mathrm{e}^{E_{1,1} / k T_{\text {rot }}} Q\left(T_{\text {rot }}\right)$,

where $g_{1,1}$ is the statistical weight, $E_{1,1}$ is the energy of the $\mathrm{NH}_{3}(1,1)$ line, $k$ is the Boltzmann constant, $T_{\text {rot }}$ is the rotational temperature, and $Q\left(T_{\text {rot }}\right)$ is the partition function. Expanding the partition function, $Q\left(T_{\text {rot }}\right)=\sum_{i} g_{i} \mathrm{e}^{-E_{i} / k T_{\text {rot }}}$ over $J$ and $K$ energy levels, where $J$ and $K$ are the transition quantum numbers (Ungerechts et al. 1986),

$N\left(\mathrm{NH}_{3}\right)=N\left[\mathrm{NH}_{3}(1,1)\right]\left[\frac{1}{3} \mathrm{e}^{+23.26 / T_{\text {rot }}}+1+\frac{5}{3} \mathrm{e}^{-41.18 / T_{\text {rot }}} \ldots\right]$,

where $T_{\text {rot }}$ is the median rotational temperature of $12.7 \mathrm{~K}$, and $N\left[\mathrm{NH}_{3}(1,1)\right]$ follows from Eq. (B.4).

We calculated the $\mathrm{H}_{2}$ column density by converting from $\mathrm{NH}_{3}$ using an abundance of $\left[\mathrm{NH}_{3}\right] /\left[\mathrm{H}_{2}\right] \sim 3 \times 10^{-8}$ (Harju et al. 1993). The final $\mathrm{H}_{2}$ column density map is shown in Fig. 5 b. 


\section{Appendix C: Deprojected filaments}
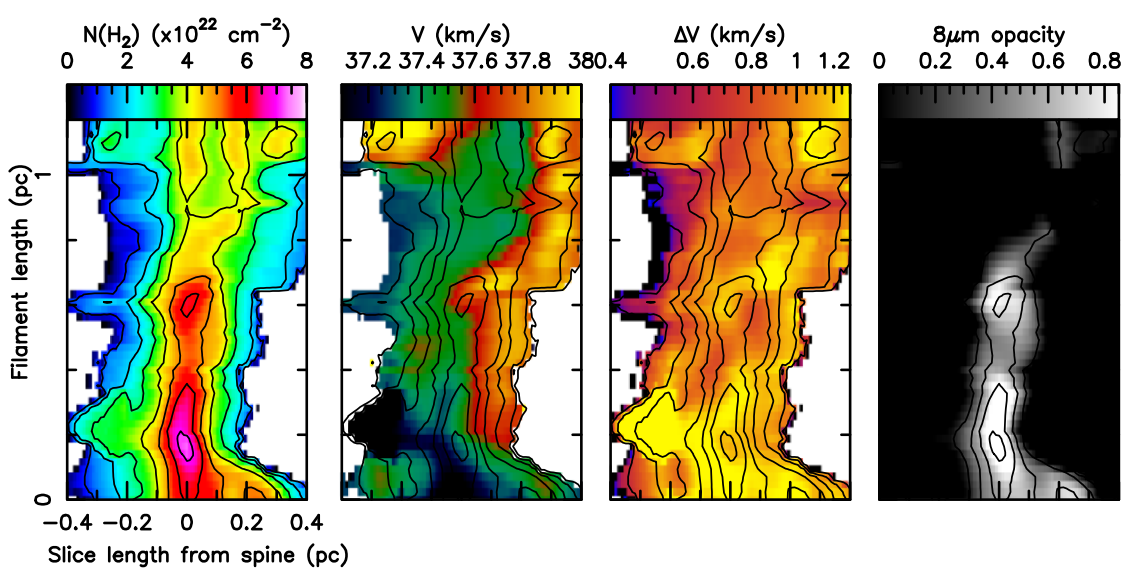

Core IDs

from spine (pc)

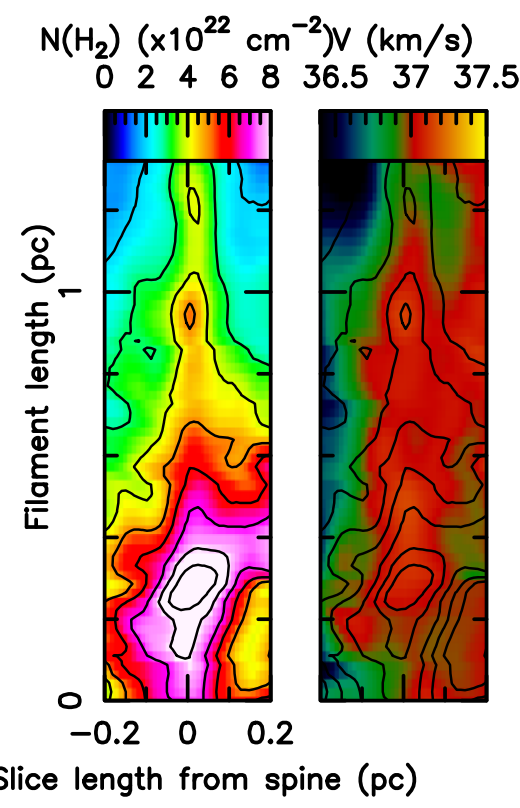

$\Delta V(\mathrm{~km} / \mathrm{s})$

$8 \mu \mathrm{m}$ opacity

Core IDs

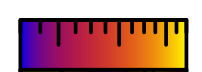

00.20.40.6.8
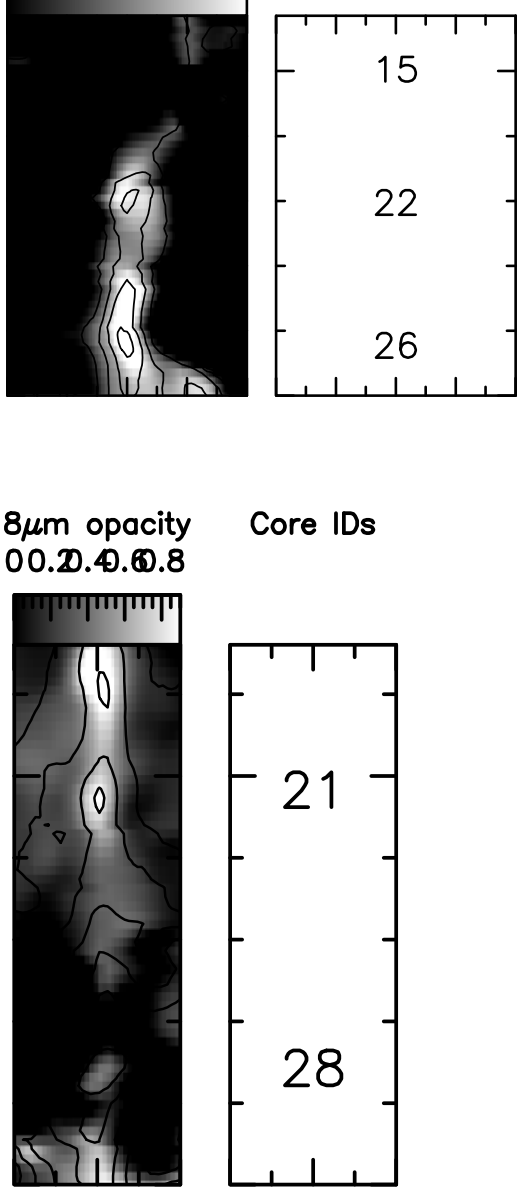

Slice length from spine (pc)

Fig. C.1. Deprojected views of the south-east (top) and north (bottom) filaments. The north filament was restricted to $0.2 \mathrm{pc}$ either side of the spine due to contamination from the north-west and north-east filaments. The filament length is plotted on the $y$-axis, while the length of the radial slices from the central spine pixel in plotted on the $x$-axis. In both sub-figures (from the left), the first panel shows the $\mathrm{H}_{2}$ column density in units of $10^{22} \mathrm{~cm}^{-2}$, the second panel shows the line-of-sight velocity in $\mathrm{km} \mathrm{s}^{-1}$, the third panel shows the velocity width in $\mathrm{km} \mathrm{s} \mathrm{s}^{-1}$, the fourth panel shows the opacity derived from the $8 \mu \mathrm{m}$ Spitzer emission, whilst the final panel labels the ID number of the identified cores. Contours in each panel are of the column density, from $1 \times 10^{22} \mathrm{~cm}^{-2}$ to $11 \times 10^{22} \mathrm{~cm}^{-2}$, spaced by $1 \times 10^{22} \mathrm{~cm}^{-2}$.

In Fig. C.1 we show the deprojected views of the south-east and north filaments (in the top and bottom panels, respectively).
It is clear that there are radial velocity gradients present in both, stronger in the south-east. 


\section{Appendix D: GBT data}
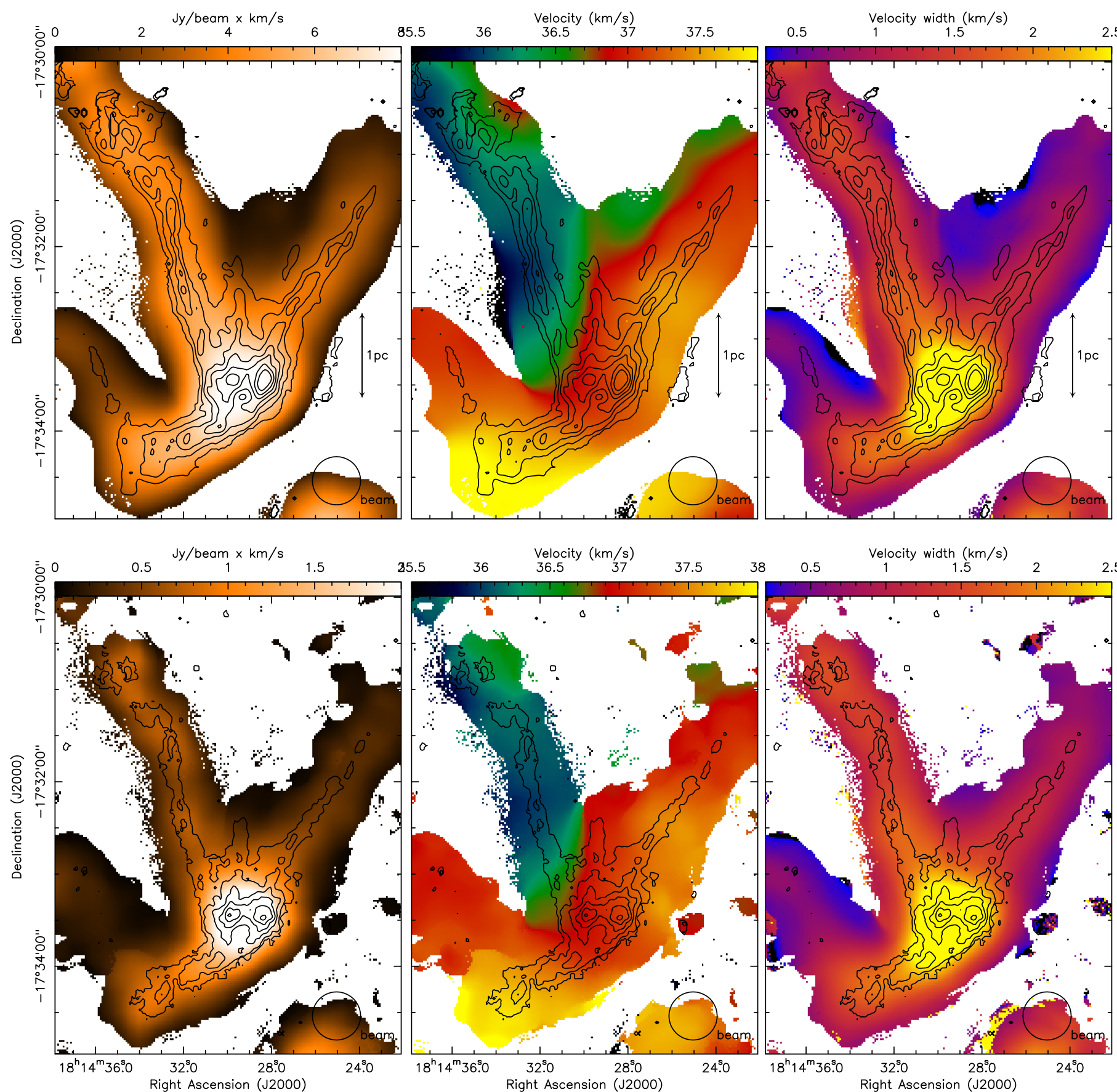

Fig. D.1. Integrated intensity (first column), centroid velocity (middle column) and velocity dispersion (right column) of the $\mathrm{NH}_{3}(1,1)$ transition (top row) and $\mathrm{NH}_{3}(2,2)$ transition (bottom row) of the GBT data. The data has been regridded to the JVLA pixel size. The $\mathrm{NH}_{3}(1,1)$ data was masked to $3 \sigma$, while the weaker $\mathrm{NH}_{3}(2,2)$ data was masked to $2 \sigma$. Contours in the top row correspond to the integrated intensity of the combined JVLA and GBT $\mathrm{NH}_{3}(1,1)$ data in steps of $0.04 \mathrm{Jy} \mathrm{beam}^{-1} \times \mathrm{km} \mathrm{s}^{-1}$, from $0.05 \mathrm{Jy} \mathrm{beam}^{-1} \times \mathrm{km} \mathrm{s}^{-1}$ to $0.29 \mathrm{Jy} \mathrm{beam}^{-1} \times \mathrm{km} \mathrm{s}^{-1}$, while contours in the bottom row correspond to the integrated intensity of the combined $\mathrm{NH}_{3}(2,2)$ data in steps of $0.008 \mathrm{Jy} \mathrm{beam}^{-1} \times \mathrm{km} \mathrm{s}^{-1}$, from $0.008 \mathrm{Jy} \mathrm{beam}^{-1} \times \mathrm{km} \mathrm{s}^{-1}$ to $0.096 \mathrm{Jy}_{\text {beam }}^{-1} \times \mathrm{km} \mathrm{s}^{-1}$. Beam information is plotted in the bottom right corner of each panel, while the scale of $1 \mathrm{pc}$ is plotted in each panel of the top row.

In Fig. D.1 we show the $\mathrm{NH}_{3}(1,1)$ and $\mathrm{NH}_{3}(2,2)$ integrated intensity, centroid line-of-sight velocity and velocity width of the GBT data. 


\section{Appendix E: Characterising radial column density profiles}
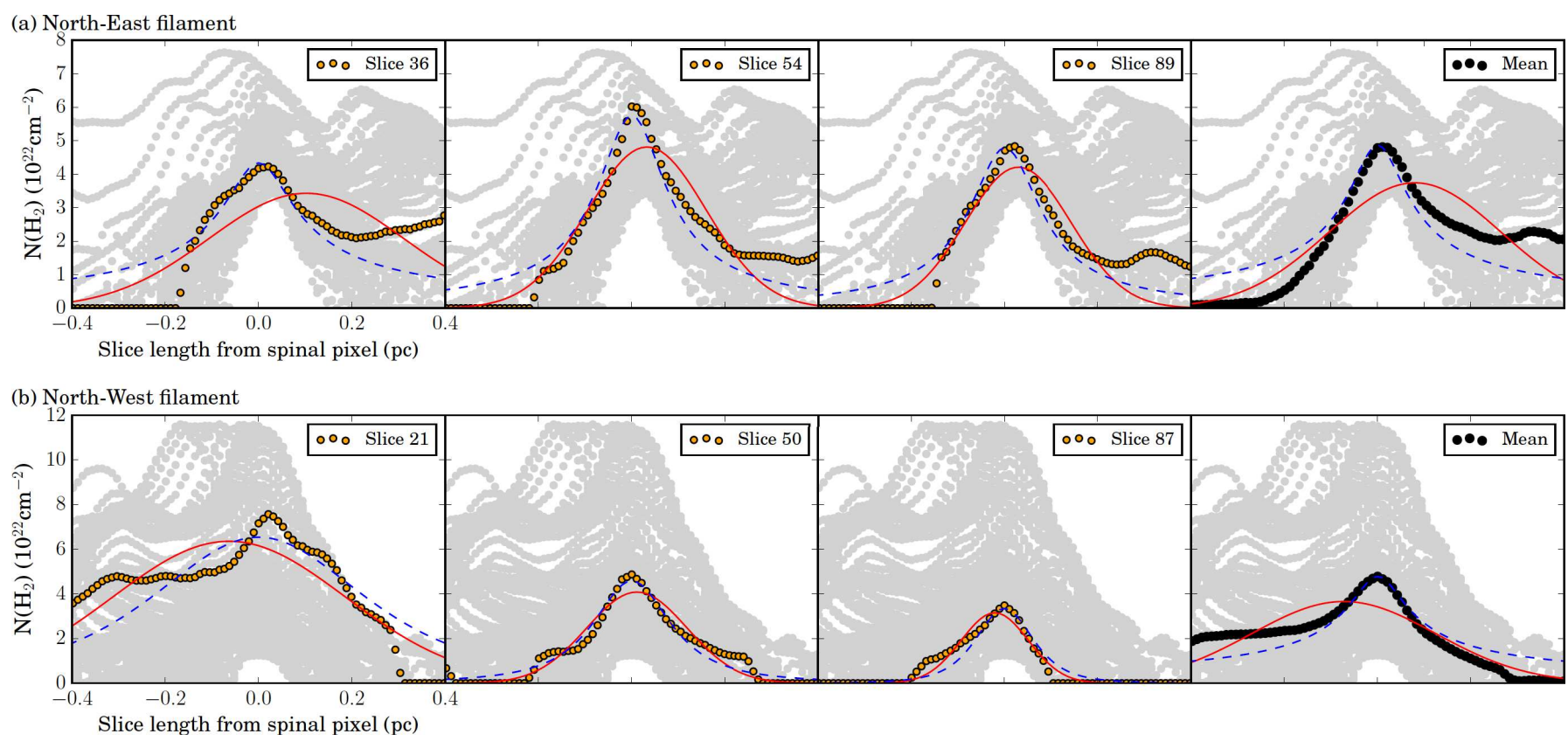

Fig. E.1. Results of fitting Gaussian (red line) and Plummer (blue dashed line) profiles to individual radial column density slices (orange circles in the first, second and third panels of each row) and the mean radial column density profile (black circles in the fourth panel of each row) of (a) the north-east filament (top row) and (b) the north-west filament (bottom row). The grey points in the background of each panel plots all of the radial column density slices simultaneously. Plummer profiles may on occasion well describe the inner regions of the filaments only, whilst Gaussian profiles fail to fit any of the distributions. Gaussian profiles are only found to reasonably fit some of the data once the fitting range is restricted to the inner portion of the filament.

To characterise the SDC13 filaments, we proceeded to fit each radial column density slice (found in Fig. 13) with Gaussian and Plummer (Whitworth \& Ward-Thompson 2001) profiles, and intended to find the function that best described the observed structure. Examples of such fits are shown in Fig. E.1. Looking at the mean profiles of the north-east and north-west filaments (the fourth panels of Fig. E.1), it is clear that they are not well described by neither Plummer nor Gaussian fits. This trend continues when considering individual column density slices along the filaments (shown in the first three panels of Fig. E.1). Although a Plummer may reasonably well describe slice 50 in the north-west filament (and perhaps even the centre-most portion of slice 54 in the north-east filament) the extended emission in the majority of slices renders fitting them with Gaussian or
Plummer profiles inappropriate. We have evaluated further the impact of constraining the Gaussian fit parameters on the goodness of the fit itself. While setting the peak position of the Gaussian profiles to lie on the spine (i.e. $x=0$ ) does not improve the produced fits, restricting the fitting to the inner portion of the filaments does result in better Gaussian fits of the central portion of the filament. However, filament properties (width and line mass) derived from such fits are very much dependent on the exact fitting range used. Such properties calculated from Trapezoidal integration of the asymmetrical profiles (as shown in Table 3 and Fig. 14) are less affected by such restrictions, and therefore more robust. Characterising filaments is fraught with difficulty, and caution must be observed whilst deriving global properties from their mean profiles (e.g. Panopoulou et al. 2017). 


\section{Appendix F: Derivation of the analytical expression of the virial ratio}

Consider a uniform density filament fragmenting at the length scale equal to the separation of a core, $\lambda_{\text {core }}$. In an idealised scenario, energy is conserved during collapse whilst fragmentation is happening. However, there is a fraction of energy converted from gravitational potential energy $\left(E_{g}\right)$ into kinetic energy $\left(E_{k}\right)$ at the time the filament fragmentation starts. This conversion may be expressed in the virial equation as an efficiency $\epsilon$, where if no energy is converted, $\epsilon=0$. If energy conversion does occur, this can be expressed as a fractional increase in $E_{k}$, expressed as $E_{k}=E_{k, 0}+\Delta E_{k}$, where $E_{k, 0}$ is the initial kinetic energy of the fragmenting core before collapse. This fractional increase in kinetic energy can be expressed as $\Delta E_{k}=\epsilon\left(E_{g}-E_{g, 0}\right)$, where $E_{g, 0}$ is the initial gravitational potential energy. Substitution into the standard virial ratio of $\alpha_{\mathrm{vir}}=2 E_{k} / E_{g}$ gives:

$\alpha_{\mathrm{vir}}=\frac{2 E_{k, 0}+2 \epsilon\left(E_{g, 0}-E_{g}\right)}{E_{g}}$,

and simplifying gives:

$\alpha_{\text {vir }}=2 \frac{E_{k, 0}}{\left|E_{g}\right|}+2 \epsilon\left(1-\frac{\left|E_{g, 0}\right|}{\left|E_{g}\right|}\right)$.

The energy terms may be expressed as:

$E_{k, 0}=\frac{3}{2} M a_{\mathrm{eff}, 0}^{2}$ where $a_{\mathrm{eff}, 0}$ is the $1 \mathrm{D}$ velocity dispersion, and:

$E_{g}=-\beta \frac{G M^{2}}{R}$,

where $\beta=\frac{3-k_{\rho}}{5-2 k_{\rho}}$ is a factor depending on the power law index $k_{\rho}$ of the density profile of collapsing cores, and:

$E_{g, 0}=-\beta_{0} \frac{G M^{2}}{R_{0}}$

where $\beta_{0}$ and $R_{0}$ are the initial values of $\beta$ and $R$ before the onset of collapse. Making these substitutions gives:

$\alpha_{\mathrm{vir}}=\frac{3}{\beta G} \frac{R a_{\mathrm{eff}, 0}^{2}}{M}+2 \epsilon\left(1-\frac{\beta_{0}}{\beta} \frac{R}{R_{0}}\right)$.

One may rewrite $R_{o}$ as $\lambda_{\text {core }} / 2$ under the assumption that all cores are initially their separation in size, and $\beta_{0}=3 / 5$ under the assumption that the filament is initially uniform in density (i.e. $k_{\rho}=0$ ) before the onset of collapse. Making these assumptions simplifies the equation to:

$\alpha_{\mathrm{vir}}=\frac{3}{\beta G} \frac{R a_{\mathrm{eff}, 0}^{2}}{M}+2 \epsilon\left(1-\frac{6}{5 \beta} \frac{R}{\lambda_{\text {core }}}\right)$. 